Comment. Math. Helv. 72 (1997) 285-315

(C) 1997 Birkhäuser Verlag, Basel

$0010-2571 / 97 / 020285-31 \$ 1.50+0.20 / 0$

Commentarii Mathematici Helvetici

\title{
The braid monodromy of plane algebraic curves and hyperplane arrangements
}

\author{
Daniel C. Cohen and Alexander I. Suciu*
}

\begin{abstract}
To a plane algebraic curve of degree $n$, Moishezon associated a braid monodromy homomorphism from a finitely generated free group to Artin's braid group $B_{n}$. Using Hansen's polynomial covering space theory, we give a new interpretation of this construction. Next, we provide an explicit description of the braid monodromy of an arrangement of complex affine hyperplanes, by means of an associated "braided wiring diagram." The ensuing presentation of the fundamental group of the complement is shown to be Tietze-I equivalent to the RandellArvola presentation. Work of Libgober then implies that the complement of a line arrangement is homotopy equivalent to the 2-complex modeled on either of these presentations. Finally, we prove that the braid monodromy of a line arrangement determines the intersection lattice. Examples of Falk then show that the braid monodromy carries more information than the group of the complement, thereby answering a question of Libgober.
\end{abstract}

Mathematics Subject Classification (1991). Primary 14H30, 20F36, 52B30; Secondary 05B35, 32S25, 57M05.

Keywords. Braid monodromy, plane curve, hyperplane arrangement, fundamental group, polynomial cover, braid group, wiring diagram, intersection lattice.

\section{Introduction}

\section{1 .}

Let $\mathcal{C}$ be an algebraic curve in $\mathbb{C}^{2}$. In the 1930 's, Zariski commissioned van Kampen to compute the fundamental group of the complement, $\pi_{1}\left(\mathbb{C}^{2} \backslash \mathcal{C}\right)$. The algorithm for doing this was developed in [vK]. Refinements of van Kampen's algorithm were given by Chisini in the 50's, and Chéniot, Abelson, and Chang in the 70's. In the early 80 's, Moishezon [Mo] introduced the notion of braid monodromy, which he used to recover van Kampen's presentation. Finally, Libgober [L1] showed that the

*The second author was partially supported by NSF. grant DMS-9504833, and an RSDF grant from Northeastern University. 
2-complex associated to the braid monodromy presentation is homotopy equivalent to $\mathbb{C}^{2} \backslash \mathcal{C}$.

Let $\mathcal{A}$ be an arrangement of hyperplanes in $\mathbb{C}^{\ell}$. In the early 80's, Randell [R1] found an algorithm for computing the fundamental group of the complement, $\pi_{1}\left(\mathbb{C}^{\ell} \backslash \mathcal{A}\right)$, when $\mathcal{A}$ is the complexification of a real arrangement. Salvetti [S1] subsequently found a regular cell complex that is a deformation retract of the complement of such an arrangement. When $\ell=2$, Falk [Fa] proved that the 2 -complex associated to the Randell presentation is homotopy equivalent to $\mathbb{C}^{2} \backslash \mathcal{A}$ by showing that it is homotopy equivalent to Salvetti's complex. The braid monodromy of a complexified real arrangement was determined by Salvetti [S2], Hironaka [Hir], and Cordovil and Fachada $[\mathrm{CF}]$, [Cor]. An algorithm for computing the fundamental group of an arbitrary complex arrangement was found by Arvola [Ar] (see also Orlik and Terao $[\mathrm{OT}]$, and see Dung and $\mathrm{Ha}[\mathrm{DH}]$ for another method).

In this paper, we present a unified view of these two subjects, extending several of the aforementioned results. In particular, we give in 5.3 an algorithm for finding the (pure) braid monodromy of an arbitrary arrangement $\mathcal{A}$ of complex lines in $\mathbb{C}^{2}$. Furthermore, we show in Theorem 6.4 that the corresponding presentation of $\pi_{1}\left(\mathbb{C}^{2} \backslash \mathcal{A}\right)$ is equivalent to the Randell-Arvola presentation. We also strengthen Falk's result, by showing that the 2-complex modeled on the Arvola presentation is homotopy equivalent to $\mathbb{C}^{2} \backslash \mathcal{A}$.

The determination of the braid monodromy of an arrangement $\mathcal{A}$ is facilitated by use of a braided wiring diagram associated to $\mathcal{A}$, a natural generalization of a combinatorial notion of Goodman [Go]. For a real arrangement, Cordovil and Fachada have shown that the braid monodromy of the complexification is determined by an associated (unbraided) wiring diagram, and have defined the braid monodromy of an abstract wiring diagram. Hironaka's technique may also be applied in this generality. The algorithm presented here generalizes both these methods.

\section{2.}

Before specializing to arrangements, we present a new interpretation of the process by which the braid monodromy of a curve $\mathcal{C}$ is defined. This follows in spirit the approach in [L1], but uses a self-contained argument based on Hansen's theory of polynomial covering maps, [H1], [H2]. Given a simple Weierstrass polynomial $f: X \times \mathbb{C} \rightarrow \mathbb{C}$ of degree $n$, we consider the space $Y=X \times \mathbb{C} \backslash\{f(x, z)=$ $0\}$. In Theorem 2.3, we show that the projection $p=\left.\operatorname{pr}_{1}\right|_{Y}: Y \rightarrow X$ is a fiber bundle map, with structure group the braid group $B_{n}$, and monodromy the homomorphism from $\pi_{1}(X)$ to $B_{n}$ induced by the coefficient map of $f$.

This result is applied in the situation where $f$ defines a plane curve $\mathcal{C}$, and $X=\mathbb{C} \backslash\left\{y_{1}, \ldots, y_{s}\right\}$ is the set of regular values of a generic linear projection. The braid monodromy of $\mathcal{C}$ is simply the coefficient homomorphism, $\alpha: F_{s} \rightarrow B_{n}$. This map depends on choices of projection, generating curves, and basepoints. 
However, the braid-equivalence class of the monodromy - the double coset $[\alpha] \in$ $B_{s} \backslash \operatorname{Hom}\left(F_{s}, B_{n}\right) / B_{n}$, where $B_{s}$ acts on the left by the Artin representation, and $B_{n}$ acts on the right by conjugation - is uniquely determined by $\mathcal{C}$.

For a line arrangement $\mathcal{A}$, changes in the various choices noted above give rise to changes in the associated braided wiring diagram $\mathcal{W}$. These, and other, "Markov moves" do not affect the braid monodromy. In practice, the braided wiring diagram of a given arrangement may be simplified via these moves. Such simplifications, together with use of the braid relations, make the braid monodromy presentation of the group of a complex arrangement accessible. Furthermore, braided wiring diagrams associated to arrangements which are lattice-isotopic in the sense of Randell [R2] are related by Markov moves. A combinatorial characterization of this fact remains to be determined. Such a characterization, suggested for (unbraided) wiring diagrams by Björner, Las Vergnas, Sturmfels, White, and Ziegler in [BLSWZ], Exercise 6.12, would likely lead to the development of a Jones-type polynomial for arrangements.

The braid monodromy is also useful in defining Alexander-type invariants of plane algebraic curves. Given a curve $\mathcal{C}$ with braid monodromy $\alpha: F_{s} \rightarrow B_{n}$, one may consider a representation $\theta: B_{n} \rightarrow \mathrm{GL}(N, R)$, and compute the module of coinvariants of $\theta \circ \alpha$. As noted by Libgober in [L3], the $R$-module $A_{\theta}(\mathcal{C})=$ $H_{0}\left(F_{s} ; R_{\theta \circ \alpha}^{N}\right)$ depends only on the equisingular isotopy class of $\mathcal{C}$ (and on $\theta$ ). When $\theta$ is the Burau representation, $A_{\theta}(\mathcal{C})$ equals the Alexander module, and thus depends only on $\pi_{1}\left(\mathbb{C}^{2} \backslash \mathcal{C}\right)$. For other representations of the braid group, such as the generalized Burau representations of [CS1], the module $A_{\theta}(\mathcal{C})$ is more likely to be a homeomorphism-type (rather than homotopy-type) invariant of the complement, see the discussion in 1.3, and section 7. For a detailed analysis of Alexander invariants of hyperplane arrangements, based on the techniques developed in this paper, we refer to [CS2].

\section{3.}

In general, the braid monodromy of a plane algebraic curve depends not only on the number and type of singularities, but on the relative positions of the singularities as well. A famous example of Zariski [Z1], [Z2] consists of two sextics, both with six cusps, one with all cusps on a conic, the other not. Explicit braid monodromy generators for these curves were given by Rudolph $[\mathrm{Ru}]$, Example 3. As shown by Zariski, the two curves have distinct fundamental groups. Further information concerning such "Zariski couples" may be found in $[\mathrm{A}-\mathrm{B}]$. An example of a different nature is given in 7.4. There, the two sextics have the same number of double points (9) and triple points (2); their fundamental groups are isomorphic, but, nevertheless, their braid monodromies are not braid-equivalent.

The above example provides an affirmative answer to a question of Libgober, who raised the possibility in [L3] that the braid monodromy of a plane algebraic curve which is transverse to the line at infinity carries more information than the 
fundamental group of the complement. The sextics in 7.4 define arrangements, originally studied by Falk $[\mathrm{Fa}]$, with distinct lattices. This explains the difference in the braid monodromies: In Theorem 7.2, we show that the braid-equivalence class of the monodromy of an arrangement determines the lattice. On the other hand, as Falk demonstrated with these examples, the homotopy type of the complement of an arrangement does not determine the lattice. However, as noted by Jiang and Yau [JY], the complements of these arrangements are not homeomorphic. This, and other evidence, suggests that the braid monodromy of a curve is more closely tied to the homeomorphism type of the complement (or even to the ambient homeomorphism type of the curve) than to the fundamental group of the complement.

In the other direction, using classical configurations of MacLane [MacL], Rybnikov $[\mathrm{Ry}]$ constructs complex arrangements with isomorphic lattices and distinct fundamental groups. It follows that the lattice of a complex arrangement does not determine the braid monodromy. We provide another illustration of this phenomenon. In Theorem 3.9, we show that complex conjugate algebraic curves have equivalent braid monodromies. However, we show in 7.7 that the monodromies of a pair of conjugate arrangements associated to MacLane's configurations are not braid-equivalent, despite the fact that these arrangements have isomorphic lattices and groups (and, in fact, diffeomorphic complements).

It is not known whether the lattice of a real arrangement determines the braid monodromy of its complexification. A result along these lines may be found in $[\mathrm{CF}]$. There, the image in the pure braid group of the braid monodromy of a wiring diagram $\mathcal{W}$ is called the braid monodromy group of $\mathcal{W}$. Cordovil and Fachada show that wiring diagrams which determine identical matroids give rise to equal braid monodromy groups. This result is not as widely applicable as it may appear. In 7.5, we consider arrangements with isomorphic (oriented) matroids and homeomorphic complements. Their monodromies are braid-equivalent, but the associated braid monodromy groups are not conjugate subgroups of the pure braid group.

Conventions. Given elements $x$ and $y$ in a group $G$, we will write $x^{y}=y^{-1} x y$ and $[x, y]=x y x^{-1} y^{-1}$. Also, we will denote by $\operatorname{Aut}(G)$ the group of right automorphisms of $G$, with multiplication $\alpha \cdot \beta=\beta \circ \alpha$.

\section{Acknowledgements}

We would like to thank Mike Falk for useful conversations, and for sharing with us his unpublished work with Bernd Sturmfels, as well as that of John Keaty. 


\section{Polynomial covers and $B_{n}$-bundles}

We begin by reviewing polynomial covering maps. These were introduced by Hansen in [H1], and studied in detail in his book [H2], which, together with Birman's book [Bi], is our basic reference for this section. We then consider bundles whose structure group is Artin's braid group $B_{n}$, and relate them to polynomial $n$-fold covers.

\subsection{Polynomial covers}

Let $X$ be a path-connected space that has the homotopy type of a CW-complex. A simple Weierstrass polynomial of degree $n$ is a map $f: X \times \mathbb{C} \rightarrow \mathbb{C}$ given by

$$
f(x, z)=z^{n}+\sum_{i=1}^{n} a_{i}(x) z^{n-i},
$$

with continuous coefficient maps $a_{i}: X \rightarrow \mathbb{C}$, and with no multiple roots for any $x \in X$. Given such $f$, the restriction of the first-coordinate projection map $\operatorname{pr}_{1}: X \times \mathbb{C} \rightarrow X$ to the subspace

$$
E=E(f)=\{(x, z) \in X \times \mathbb{C} \mid f(x, z)=0\}
$$

defines an $n$-fold cover $\pi=\pi_{f}: E \rightarrow X$, the polynomial covering map associated to $f$.

Since $f$ has no multiple roots, the coefficient map $a=\left(a_{1}, \ldots, a_{n}\right): X \rightarrow \mathbb{C}^{n}$ takes values in the complement of the discriminant set, $B^{n}=\mathbb{C}^{n} \backslash \Delta_{n}$. Over $B^{n}$, there is a canonical $n$-fold polynomial covering map $\pi_{n}=\pi_{f_{n}}: E\left(f_{n}\right) \rightarrow B^{n}$, determined by the Weierstrass polynomial $f_{n}(x, z)=z^{n}+\sum_{i=1}^{n^{n}} x_{i} z^{n-i}$. Clearly, the polynomial cover $\pi_{f}: E(f) \rightarrow X$ is the pull-back of $\pi_{n}: E\left(f_{n}\right) \rightarrow B^{n}$ along the coefficient map $a: X \rightarrow B^{n}$.

This can be interpreted on the level of fundamental groups as follows. The fundamental group of the configuration space, $B^{n}$, of $n$ unordered points in $\mathbb{C}$ is the group, $B_{n}$, of braids on $n$ strands. The map $a$ determines the coefficient homomorphism $\alpha=a_{*}: \pi_{1}(X) \rightarrow B_{n}$, unique up to conjugacy. One may characterize polynomial covers as those covers $\pi: E \rightarrow X$ for which the characteristic homomorphism to the symmetric group, $\chi: \pi_{1}(X) \rightarrow \Sigma_{n}$, factors through the canonical surjection $\tau_{n}: B_{n} \rightarrow \Sigma_{n}$ as $\chi=\tau_{n} \circ \alpha$.

Now assume that the simple Weierstrass polynomial $f$ is completely solvable, that is, factors as

$$
f(x, z)=\prod_{i=1}^{n}\left(z-b_{i}(x)\right),
$$

with continuous roots $b_{i}: X \rightarrow \mathbb{C}$. Since the Weierstrass polynomial $f$ is simple, the root map $b=\left(b_{1}, \ldots, b_{n}\right): X \rightarrow \mathbb{C}^{n}$ takes values in the complement, 
$P^{n}=\mathbb{C}^{n} \backslash \mathcal{A}_{n}$, of the braid arrangement $\mathcal{A}_{n}=\left\{\operatorname{ker}\left(w_{i}-w_{j}\right)\right\}_{1 \leq i<j \leq n}$. Over $P^{n}$, there is a canonical $n$-fold covering map, $q_{n}=\pi_{Q_{n}}: E\left(Q_{n}\right) \rightarrow P^{n}$, determined by the Weierstrass polynomial $Q_{n}(w, z)=\left(z-w_{1}\right) \cdots\left(z-w_{n}\right)$. Evidently, the cover $\pi_{f}: E \rightarrow X$ is the pull-back of $q_{n}: E\left(Q_{n}\right) \rightarrow P^{n}$ along the root map $b: X \rightarrow P^{n}$.

The fundamental group of the configuration space, $P^{n}$, of $n$ ordered points in $\mathbb{C}$ is the group, $P_{n}=\operatorname{ker} \tau_{n}$, of pure braids on $n$ strands. The map $b$ determines the root homomorphism $\beta=b_{*}: \pi_{1}(X) \rightarrow P_{n}$, unique up to conjugacy. The polynomial covers which are trivial covers (in the usual sense) are precisely those for which the coefficient homomorphism factors as $\alpha=\iota_{n} \circ \beta$, where $\iota_{n}: P_{n} \rightarrow B_{n}$ is the canonical injection.

\section{2. $B_{n}$-Bundles}

The group $B_{n}$ may be realized as the mapping class group $\mathfrak{M}_{0,1}^{n}$ of orientationpreserving diffeomorphisms of the disk $D^{2}$, permuting a collection of $n$ marked points. Upon identifying $\pi_{1}\left(D^{2} \backslash\{n\right.$ points $\left.\}\right)$ with the free group $F_{n}$, the action of $B_{n}$ on $\pi_{1}$ yields the Artin representation, $\alpha_{n}: B_{n} \rightarrow \operatorname{Aut}\left(F_{n}\right)$. As shown by Artin, this representation is faithful. Hence, we may - and often will - identify a braid $\theta \in B_{n}$ with the corresponding braid automorphism, $\alpha_{n}(\theta) \in \operatorname{Aut}\left(F_{n}\right)$.

Now let $f: X \times \mathbb{C} \rightarrow \mathbb{C}$ be a simple Weierstrass polynomial. Let $\pi_{f}: E(f) \rightarrow X$ be the corresponding polynomial $n$-fold cover, and $a: X \rightarrow B^{n}$ the coefficient map. Consider the complement

$$
Y=Y(f)=X \times \mathbb{C} \backslash E(f),
$$

and let $p=p_{f}: Y(f) \rightarrow X$ be the restriction of $\operatorname{pr}_{1}: X \times \mathbb{C} \rightarrow X$ to $Y$.

Theorem 2.3. The map $p: Y \rightarrow X$ is a locally trivial bundle, with structure group $B_{n}$ and fiber $\mathbb{C}_{n}=\mathbb{C} \backslash\{n$ points $\}$. Upon identifying $\pi_{1}\left(\mathbb{C}_{n}\right)$ with $F_{n}$, the monodromy of this bundle may be written as $\alpha_{n} \circ \alpha$, where $\alpha=a_{*}: \pi_{1}(X) \rightarrow B_{n}$ is the coefficient homomorphism.

Moreover, if $f$ is completely solvable, the structure group reduces to $P_{n}$, and the monodromy factors as $\alpha_{n} \circ \iota_{n} \circ \beta$, where $\beta=b_{*}: \pi_{1}(X) \rightarrow P_{n}$ is the root homomorphism.

Proof. We first prove the theorem for the configuration spaces, and their canonical Weierstrass polynomials. Start with $X=P^{n}, f=Q_{n}$, and the canonical cover $q_{n}: E\left(Q_{n}\right) \rightarrow P^{n}$. Clearly, $Y\left(Q_{n}\right)=\mathbb{C}^{n+1} \backslash E\left(Q_{n}\right)$ is equal to the configuration space $P^{n+1}$. Let $\rho_{n}=p_{Q_{n}}: P^{n+1} \rightarrow P^{n}$ be the restriction of $\operatorname{pr}_{1}: \mathbb{C}^{n} \times \mathbb{C} \rightarrow \mathbb{C}^{n}$. As shown by Fadell and Neuwirth [FN], this is a bundle map, with fiber $\mathbb{C}_{n}$, and monodromy the restriction of the Artin representation to $P_{n}$.

Next, consider $X=B^{n}, f=f_{n}$, and the canonical cover $\pi_{n}: E\left(f_{n}\right) \rightarrow$ $B^{n}$. Forgetting the order of the points defines a covering projection from the ordered to the unordered configuration space, $\kappa_{n}: P^{n} \rightarrow B^{n}$. In coordinates, 
$\kappa_{n}\left(w_{1}, \ldots, w_{n}\right)=\left(x_{1}, \ldots, x_{n}\right)$, where $x_{i}=(-1)^{i} s_{i}\left(w_{1}, \ldots, w_{n}\right)$, and $s_{i}$ are the elementary symmetric functions. By Vieta's formulas, we have

$$
Q_{n}(w, z)=f_{n}\left(\kappa_{n}(w), z\right)
$$

Let $Y^{n+1}=Y\left(f_{n}\right)$ and $p_{n}=p_{f_{n}}: Y^{n+1} \rightarrow B^{n}$. By the above formula, we see that $\kappa_{n} \times$ id $: P^{n} \times \mathbb{C} \rightarrow B^{n} \times \mathbb{C}$ restricts to a map $\bar{\kappa}_{n+1}: Y\left(Q_{n}\right) \rightarrow Y\left(f_{n}\right)$, which fits into the fiber product diagram

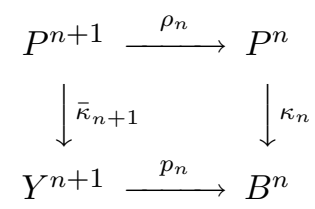

where the vertical maps are principal $\Sigma_{n}$-bundles. Since the bundle map $\rho_{n}$ : $P^{n+1} \rightarrow P^{n}$ is equivariant with respect to the $\Sigma_{n}$-actions, the map on quotients, $p_{n}: Y^{n+1} \rightarrow B^{n}$, is also a bundle map, with fiber $\mathbb{C}_{n}$, and monodromy action the Artin representation of $B_{n}$. This finishes the proof in the case of the canonical Weierstrass polynomials over configuration spaces.

Now let $f: X \times \mathbb{C} \rightarrow \mathbb{C}$ be an arbitrary simple Weierstrass polynomial. We then have the following cartesian square:



In other words, $p: Y \rightarrow X$ is the pullback of the bundle $p_{n}: Y^{n+1} \rightarrow B^{n}$ along the coefficient map $a$. Thus, $p$ is a bundle map, with fiber $\mathbb{C}_{n}$, and monodromy representation $\alpha_{n} \circ \alpha$. When $f$ is completely solvable, the bundle $p: Y \rightarrow X$ is the pullback of $\rho_{n}: P^{n+1} \rightarrow P^{n}$ along the root map $b$. Since $\alpha=\iota_{n} \circ \beta$, the monodromy is as claimed.

Remark 2.4. Let us summarize the above discussion of braid bundles over configuration spaces. From the Fadell-Neuwirth theorem, it follows that $P^{n}$ is a $K\left(P_{n}, 1\right)$ space. Since the pure braid group is discrete, the classifying $P_{n}$-bundle (in the sense of Steenrod) is the universal cover $\widetilde{P}^{n} \rightarrow P^{n}$. We considered two bundles over $P^{n}$, both associated to this one:

(i) $q_{n}: E\left(Q_{n}\right) \rightarrow X^{n}$, by the trivial representation of $P_{n}$ on $\{1, \ldots, n\}$;

(ii) $\rho_{n}: P^{n+1} \rightarrow P^{n}$, by the (geometric) Artin representation of $P_{n}$ on $\mathbb{C}_{n}$. Since $B^{n}$ is covered by $P^{n}$, it is a $K\left(B_{n}, 1\right)$ space, and the classifying $B_{n}$-bundle is $\widetilde{B}^{n} \rightarrow B^{n}$. There were three bundles over $B^{n}$ that we mentioned, all associated to this one: 
(iii) $\kappa_{n}: X^{n} \rightarrow B^{n}$, by the canonical surjection $\tau_{n}: B_{n} \rightarrow \Sigma_{n}$;

(iv) $\pi_{n}: E\left(f_{n}\right) \rightarrow B^{n}$, by the above, followed by the permutation representation of $\Sigma_{n}$ on $\{1, \ldots, n\}$

(v) $p_{n}: Y^{n+1} \rightarrow B^{n}$, by the (geometric) Artin representation of $B_{n}$ on $\mathbb{C}_{n}$.

Finally, note that $\pi_{1}\left(Y^{n+1}\right)$ is isomorphic to $B_{n}^{1}=F_{n} \rtimes_{\alpha_{n}} B_{n}$, the group of braids on $n+1$ strands that fix the endpoint of the last strand, and that $Y^{n+1}$ is a $K\left(B_{n}^{1}, 1\right)$ space.

\section{The braid monodromy of a plane algebraic curve}

We are now ready to define the braid monodromy of an algebraic curve in the complex plane. The construction, based on classical work of Zariski and van Kampen, is due to Moishezon [Mo]. We follow the exposition of Libgober [L1], [L2], [L3], but interpret the construction in the context established in the previous section.

\subsection{The construction}

Let $\mathcal{C}$ be a reduced algebraic curve in $\mathbb{C}^{2}$, with defining polynomial $f$ of degree $n$. Let $\pi: \mathbb{C}^{2} \rightarrow \mathbb{C}$ be a linear projection, and let $\mathcal{Y}=\left\{y_{1}, \ldots, y_{s}\right\}$ be the set of points in $\mathbb{C}$ for which the fibers of $\pi$ contain singular points of $\mathcal{C}$, or are tangent to $\mathcal{C}$. Assume that $\pi$ is generic with respect to $\mathcal{C}$. That is, for each $k$, the line $\mathcal{L}_{k}=\pi^{-1}\left(y_{k}\right)$ contains at most one singular point $v_{k}$ of $\mathcal{C}$, and does not belong to the tangent cone of $\mathcal{C}$ at $v_{k}$, and, moreover, all tangencies are simple. Let $\mathcal{L}$ denote the union of the lines $\mathcal{L}_{k}$, and let $y_{0}$ be a basepoint in $\mathbb{C} \backslash \mathcal{Y}$. The definition of the braid monodromy of $\mathcal{C}$ depends on two observations:

(i) The restriction of the projection map, $p: \mathbb{C}^{2} \backslash \mathcal{C} \cup \mathcal{L} \rightarrow \mathbb{C} \backslash \mathcal{Y}$, is a locally trivial bundle.

Fix the fiber $\mathbb{C}_{n}=p^{-1}\left(y_{0}\right)$ and a basepoint $\hat{y}_{0} \in \mathbb{C}_{n}$. The monodromy of $\mathcal{C}$ is, by definition, the holonomy of this bundle, $\rho: \pi_{1}\left(\mathbb{C} \backslash \mathcal{Y}, y_{0}\right) \rightarrow \operatorname{Aut}\left(\pi_{1}\left(\mathbb{C}_{n}, \hat{y}_{0}\right)\right)$. Upon identifying $\pi_{1}\left(\mathbb{C} \backslash \mathcal{Y}, y_{0}\right)$ with $F_{s}$, and $\pi_{1}\left(\mathbb{C}_{n}, \hat{y}_{0}\right)$ with $F_{n}$, this can be written as $\rho: F_{s} \rightarrow \operatorname{Aut}\left(F_{n}\right)$.

(ii) The image of $\rho$ is contained in the braid group $B_{n}$ (viewed as a subgroup of Aut $\left(F_{n}\right)$ via the Artin embedding $\left.\alpha_{n}\right)$.

The braid monodromy of $\mathcal{C}$ is the homomorphism $\alpha: F_{s} \rightarrow B_{n}$ determined by $\alpha_{n} \circ \alpha=\rho$.

We shall present a self-contained proof of these two assertions, and, in the process, identify the map $\alpha$. The first assertion is well-known, and can also be 
proved by standard techniques (using blow-ups and Ehresmann's criterion-see [Di], page 123), but we find our approach sheds some light on the underlying topology of the situation.

\subsection{Braid monodromy and polynomial covers}

Let $\pi: \mathbb{C}^{2} \rightarrow \mathbb{C}^{1}$ be a linear projection, generic with respect to the given algebraic curve $\mathcal{C}$ of degree $n$. We may assume (after a linear change of variables in $\mathbb{C}^{2}$ if necessary) that $\pi=\mathrm{pr}_{1}$, the projection map onto the first coordinate. In the chosen coordinates, the defining polynomial $f$ of $\mathcal{C}$ may be written as $f(x, z)=$ $z^{n}+\sum_{i=1}^{n} a_{i}(x) z^{n-i}$. Since $\mathcal{C}$ is reduced, for each $x \notin \mathcal{Y}$, the equation $f(x, z)=0$ has $n$ distinct roots. Thus, $f$ is a simple Weierstrass polynomial over $\mathbb{C} \backslash \mathcal{Y}$, and

$$
\pi=\pi_{f}: \mathcal{C} \backslash \mathcal{C} \cap \mathcal{L} \rightarrow \mathbb{C} \backslash \mathcal{Y}
$$

is the associated polynomial $n$-fold cover.

Note that $Y(f)=((\mathbb{C} \backslash \mathcal{Y}) \times \mathbb{C}) \backslash(\mathcal{C} \backslash \mathcal{C} \cap \mathcal{L})=\mathbb{C}^{2} \backslash \mathcal{C} \cup \mathcal{L}$. By Theorem 2.3, the restriction of $\operatorname{pr}_{1}$ to $Y(f)$,

$$
p: \mathbb{C}^{2} \backslash \mathcal{C} \cup \mathcal{L} \rightarrow \mathbb{C} \backslash \mathcal{Y},
$$

is a bundle map, with structure group $B_{n}$, fiber $\mathbb{C}_{n}$, and monodromy homomorphism

$$
\alpha=a_{*}: \pi_{1}(\mathbb{C} \backslash \mathcal{Y}) \rightarrow B_{n} .
$$

This proves assertions (i) and (ii). Furthermore, we have

Theorem 3.3. The braid monodromy of a plane algebraic curve coincides with the coefficient homomorphism of the associated polynomial cover.

In the case where $\mathcal{C}=\mathcal{A}$ is an arrangement of (affine) lines in $\mathbb{C}^{2}$, more can be said. First, the critical set $\mathcal{Y}=\left\{y_{1}, \ldots, y_{s}\right\}$ consists (only) of the images under $\pi=\operatorname{pr}_{1}$ of the vertices of the arrangement. Furthermore, a defining polynomial for $\mathcal{A}$ can be written as $f(x, z)=\prod_{i=1}^{n}\left(z-\ell_{i}(x)\right)$, where each $\ell_{i}$ is a linear function in $x$. Thus, the associated polynomial cover is trivial, and the monodromy representation is

$$
\lambda=\ell_{*}: \pi_{1}(\mathbb{C} \backslash \mathcal{Y}) \rightarrow P_{n} .
$$

An explicit formula for $\lambda$ will be given in section 5 . For now, let us record the following:

Theorem 3.4. The pure braid monodromy of a line arrangement coincides with the root homomorphism of the associated (trivial) polynomial cover. 


\subsection{Braid equivalence}

The braid monodromy of a plane algebraic curve is not unique, but rather, depends on the choices made in defining it. This indeterminacy was studied by Libgober in [L2], [L3]. To make the analysis more precise, we first need a definition.

Definition 3.6. Two homomorphisms $\alpha: F_{s} \rightarrow B_{n}$ and $\alpha^{\prime}: F_{s} \rightarrow B_{n}$ are equivalent if there exist automorphisms $\psi \in \operatorname{Aut}\left(F_{s}\right)$ and $\phi \in \operatorname{Aut}\left(F_{n}\right)$ with $\phi\left(B_{n}\right) \subset B_{n}$ such that $\alpha^{\prime}(\psi(g))=\phi^{-1} \cdot \alpha(g) \cdot \phi$, for all $g \in F_{s}$. In other words, the following diagram commutes

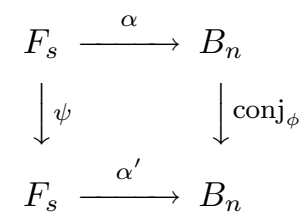

If, moreover, $\psi \in B_{s}$ and $\phi \in B_{n}$, the homomorphisms $\alpha$ and $\alpha^{\prime}$ are braidequivalent.

Theorem 3.7. The braid monodromy of a plane algebraic curve $\mathcal{C}$ is well-defined up to braid-equivalence.

Proof. First fix the generic projection. The identification $\pi_{1}(\mathbb{C} \backslash \mathcal{Y})=F_{s}$ depends on the choice of a "well-ordered" system of generators (see [Mo] or the discussion in 4.1), and any two such choices yield monodromies which differ by a braid automorphism of $F_{s}$, see [L2]. Furthermore, there is the choice of basepoints, and any two such choices yield monodromies differing by a conjugation in $B_{n}$.

Finally, one must analyze the effect of a change in the choice of generic projection. Let $\pi$ and $\pi^{\prime}$ be two such projections, with critical sets $\mathcal{Y}$ and $\mathcal{Y}^{\prime}$, and braid monodromies $\alpha: \pi_{1}(\mathbb{C} \backslash \mathcal{Y}) \rightarrow B_{n}$ and $\alpha^{\prime}: \pi_{1}\left(\mathbb{C} \backslash \mathcal{Y}^{\prime}\right) \rightarrow B_{n}$. Libgober [L3] shows that there is a homeomorphism $h: \mathbb{C} \rightarrow \mathbb{C}$, isotopic to the identity, and taking $\mathcal{Y}$ to $\mathcal{Y}^{\prime}$, for which the isomorphism $h_{*}: \pi_{1}(\mathbb{C} \backslash \mathcal{Y}) \rightarrow \pi_{1}\left(\mathbb{C} \backslash \mathcal{Y}^{\prime}\right)$ induced by the restriction of $h$ satisfies $\alpha^{\prime} \circ h_{*}=\alpha$. From the construction, we see that $h$ can be taken to be the identity outside a ball of large radius (containing $\mathcal{Y} \cup \mathcal{Y}^{\prime}$ ). Thus, once the identifications of source and target with $F_{s}$ are made, $h_{*}$ can be written as the composite of an inner automorphism of $F_{s}$ with a braid automorphism of $F_{s}$ : $h_{*}=\operatorname{conj}_{g} \circ \psi$. Trading the inner automorphism of $F_{s}$ for an inner automorphism of $B_{n}$, we obtain $\alpha^{\prime} \circ \psi=\operatorname{conj}_{\alpha^{\prime}(g)} \circ \alpha$, completing the proof.

Thus, we may regard the braid monodromy of $\mathcal{C}$ as a braid-equivalence class, i.e., as a double coset $[\alpha] \in B_{s} \backslash \operatorname{Hom}\left(F_{s}, B_{n}\right) / B_{n}$, uniquely determined by $\mathcal{C}$. In fact, it follows from [L3] that $[\alpha]$ depends only on the equisingular isotopy class of the curve. 


\subsection{Conjugate curves}

If $\mathcal{C}$ is a plane curve with defining polynomial $f=f(x, z)$ of degree $n$, let $\overline{\mathcal{C}}$ be the curve defined by the polynomial $\bar{f}$ whose coefficients are the complex conjugates of those of $f$. In other words, $\bar{f}(x, z)=\overline{f(\bar{x}, \bar{z})}$. In this subsection, we relate the braid monodromies of $\mathcal{C}$ and $\overline{\mathcal{C}}$. In general, the braid monodromies of conjugate curves are not braid-equivalent, as shown in 7.7. Nevertheless, we have the following:

Theorem 3.9. The braid monodromies of conjugate curves are equivalent.

Proof. Let $\mathcal{C}$ and $\overline{\mathcal{C}}$ be conjugate curves defined by polynomials $f$ and $\bar{f}$ of degree $n$. Choose coordinates in $\mathbb{C}^{2}$ so that $\pi=\mathrm{pr}_{1}$ is generic with respect to $\mathcal{C}$. Then $\pi$ is evidently also generic with respect to $\overline{\mathcal{C}}$. Let $\mathcal{Y}$ and $\overline{\mathcal{Y}}$ be the critical sets of $\mathcal{C}$ and $\overline{\mathcal{C}}$ with respect to this projection. Complex conjugation $\mathbb{C} \rightarrow \mathbb{C}$ restricts to a map $d: \mathbb{C} \backslash \mathcal{Y} \rightarrow \mathbb{C} \backslash \overline{\mathcal{Y}}$. Choose a basepoint $y_{0}$ with $\operatorname{Im}\left(y_{0}\right)=0$. Then $d$ induces an isomorphism $d_{*}: \pi_{1}\left(\mathbb{C} \backslash \mathcal{Y}, y_{0}\right) \rightarrow \pi_{1}\left(\mathbb{C} \backslash \overline{\mathcal{Y}}, y_{0}\right)$. Identifying these groups with $F_{s}=\left\langle x_{1}, \ldots, x_{s}\right\rangle$, we have $d_{*}=\delta_{s}$, where $\delta_{s} \in \operatorname{Aut}\left(F_{s}\right)$ is given by $\delta_{s}\left(x_{k}\right)=\left(x_{1} \cdots x_{k-1}\right) \cdot x_{k}^{-1} \cdot\left(x_{1} \cdots x_{k-1}\right)^{-1}$.

Since the discriminant locus $\Delta_{n}$ is defined by a polynomial with real coefficients, complex conjugation $\mathbb{C}^{n} \rightarrow \mathbb{C}^{n}$ restricts to a map $e: B^{n} \rightarrow B^{n}$. The induced map $\epsilon_{n}=e_{*}: B_{n} \rightarrow B_{n}$ is readily seen to be the automorphism defined on generators by $\epsilon_{n}\left(\sigma_{i}\right)=\sigma_{i}^{-1}$. As shown by Dyer and Grossman [DG], this involution generates $\operatorname{Out}\left(B_{n}\right)=\mathbb{Z}_{2}$, for $n \geq 3$.

Let $a$ and $\bar{a}$ be the coefficient maps of $f$ and $\bar{f}$ respectively. The fact that the defining polynomials of $\mathcal{C}$ and $\overline{\mathcal{C}}$ have complex conjugate coefficients may be expressed as $\bar{a} \circ d=e \circ a$. Passing to fundamental groups, we have $\bar{\alpha} \circ \delta_{s}=\epsilon_{n} \circ \alpha$. Checking that $\epsilon_{n}=\operatorname{conj}_{\delta_{n}}$ (see [DG]) completes the proof.

\section{The fundamental group of a plane algebraic curve}

We now give the braid monodromy presentation of the fundamental group of the complement of a plane algebraic curve $\mathcal{C}$. This presentation first appeared in the classical work of van Kampen and Zariski [vK], [Z2], and has been much studied since, see e.g. [Mo], [MT], [L1], [L2], [Ru], [Di].

\subsection{Braid monodromy presentation}

The homotopy exact sequence of the bundle $p: \mathbb{C}^{2} \backslash \mathcal{C} \cup \mathcal{L} \rightarrow \mathbb{C} \backslash \mathcal{Y}$ of (2) reduces to

$$
1 \rightarrow \pi_{1}\left(\mathbb{C}_{n}\right) \rightarrow \pi_{1}\left(\mathbb{C}^{2} \backslash \mathcal{C} \cup \mathcal{L}\right) \stackrel{p_{*}}{\longrightarrow} \pi_{1}(\mathbb{C} \backslash \mathcal{Y}) \rightarrow 1
$$

This sequence is split exact, with action given by the braid monodromy homo- 
morphism $\alpha$ of (3). To extract a presentation of the middle group, order the points of $\mathcal{Y}$ by decreasing real part, and pick the basepoint $y_{0}$ in $\mathbb{C} \backslash \mathcal{Y}$ with $\operatorname{Re}\left(y_{0}\right)>\max \left\{\operatorname{Re}\left(y_{k}\right)\right\}$. Choose loops $\xi_{k}:[0,1] \rightarrow \mathbb{C} \backslash \mathcal{Y}$ based at $y_{0}$, going up and above $y_{1}, \ldots, y_{k-1}$, passing around $y_{k}$ in the counterclockwise direction, and coming back the same way. Setting $x_{k}=\left[\xi_{k}\right]$, identify $\pi_{1}\left(\mathbb{C} \backslash \mathcal{Y}, y_{0}\right)$ with $F_{s}=\left\langle x_{1}, \ldots, x_{s}\right\rangle$. Similarly, identify $\pi_{1}\left(\mathbb{C}_{n}, \hat{y}_{0}\right)$ with $F_{n}=\left\langle t_{1}, \ldots, t_{n}\right\rangle$. Having done this, $\pi_{1}\left(\mathbb{C}^{2} \backslash \mathcal{C} \cup \mathcal{L}, \hat{y}_{0}\right)$ becomes identified with the semidirect product $F_{n} \rtimes_{\alpha} F_{s}$. The corresponding presentation is

$$
\pi_{1}\left(\mathbb{C}^{2} \backslash \mathcal{C} \cup \mathcal{L}\right)=\left\langle t_{1}, \ldots t_{n}, x_{1} \ldots, x_{s} \mid x_{k}^{-1} \cdot t_{i} \cdot x_{k}=\alpha\left(x_{k}\right)\left(t_{i}\right)\right\rangle .
$$

The fundamental group of the complement of the curve is the quotient of $\pi_{1}\left(\mathbb{C}^{2} \backslash \mathcal{C} \cup \mathcal{L}\right)$ by the normal closure of $F_{s}=\left\langle x_{1}, \ldots, x_{s}\right\rangle$. Thus, $\pi_{1}\left(\mathbb{C}^{2} \backslash \mathcal{C}\right)=$ $\left\langle t_{1}, \ldots, t_{n} \mid t_{i}=\alpha\left(x_{k}\right)\left(t_{i}\right)\right\rangle$. This presentation can be simplified by Tietze-II moves - eliminating redundant relations. Doing so, one obtains the braid monodromy presentation

$$
\pi_{1}\left(\mathbb{C}^{2} \backslash \mathcal{C}\right)=\left\langle t_{1}, \ldots, t_{n} \mid t_{i}=\alpha\left(x_{k}\right)\left(t_{i}\right), \quad i=j_{1}, \ldots, j_{m_{k}-1} ; \quad k=1, \ldots, s\right\rangle .
$$

If $y_{k}$ corresponds to a singular point of $\mathcal{C}$, then $m_{k}$ denotes the multiplicity of that singular point, while if $y_{k}$ corresponds to a (simple) tangency point, $m_{k}=2$. In either case, the indices $j_{1}, \ldots, j_{m_{k}-1}$ must be chosen appropriately, see [L1] and the discussions in 5.1 and 6.1 .

Let $K(\mathcal{C})$ be the 2-complex modeled on the braid monodromy presentation. There is an obvious embedding of this complex into the complement of $\mathcal{C}$. The main result of $[\mathrm{L} 1]$ is the following.

Theorem 4.2. (Libgober) The 2-complex $K(\mathcal{C})$ is a deformation retract of $\mathbb{C}^{2} \backslash \mathcal{C}$.

Remark 4.3. The group $G(\alpha)$ defined by presentation (4) is the quotient of $F_{n}$ by the normal subgroup generated by $\left\{\gamma(t) \cdot t^{-1} \mid \gamma \in \operatorname{im}(\alpha), t \in F_{n}\right\}$. In other words, $G(\alpha)$ is the maximal quotient of $F_{n}$ on which the representation $\alpha: F_{s} \rightarrow B_{n}$ acts trivially. If $\alpha^{\prime}: F_{s} \rightarrow B_{n}$ is equivalent to $\alpha$, then $G(\alpha)$ is isomorphic to $G\left(\alpha^{\prime}\right)$. Indeed, the equivalence condition $\alpha^{\prime} \circ \psi=\operatorname{conj}_{\phi} \circ \alpha$ can be written as $\phi\left(\alpha(g)(t) \cdot t^{-1}\right)=\alpha^{\prime}(\psi(g))(\phi(t)) \cdot \phi(t)^{-1}, \forall g \in F_{s}, \forall t \in F_{n}$. Thus $\phi \in \operatorname{Aut}\left(F_{n}\right)$ induces an isomorphism $\bar{\phi}: G(\alpha) \rightarrow G\left(\alpha^{\prime}\right)$.

\subsection{Braid monodromy generators}

We now make the presentation (4) more precise. First recall that the braid group $B_{n}$ has generators $\sigma_{1}, \ldots, \sigma_{n-1}$, and relations $\sigma_{i} \sigma_{i+1} \sigma_{i}=\sigma_{i+1} \sigma_{i} \sigma_{i+1}(1 \leq i<$ $n-1), \sigma_{i} \sigma_{j}=\sigma_{j} \sigma_{i}(|i-j|>1)$, see [Bi], [H2]. The Artin representation $\alpha_{n}$ : 
$B_{n} \rightarrow \operatorname{Aut}\left(F_{n}\right)$ is given by:

$$
\sigma_{i}\left(t_{j}\right)= \begin{cases}t_{i} t_{i+1} t_{i}^{-1} & \text { if } j=i \\ t_{i} & \text { if } j=i+1 \\ t_{j} & \text { otherwise. }\end{cases}
$$

For each $k=1, \ldots, s$, let $\gamma_{k} \in B_{m_{k}}<B_{n}$ be the "local monodromy" around $y_{k}$. Then

$$
\alpha\left(x_{k}\right)=\beta_{k}^{-1} \gamma_{k} \beta_{k}
$$

where $\beta_{k} \in B_{n}$ is the monodromy along the portion of $\xi_{k}$ from $y_{0}$ to just before $y_{k}$. One would like to express these braids in terms of the standard generators $\sigma_{i}$ of $B_{n}$. This may be accomplished in two steps.

Step 1. The structure of the (isolated) singularity $v_{k}$ above $y_{k}$ determines the local braid $\gamma_{k}$. This braid may be obtained from the Puiseux series expansion of the defining polynomial $f(x, z)$ of $\mathcal{C}$. This is implicit in the work of Brieskorn and Knörrer [BK] and Eisenbud and Neumann [EN].

Example 4.5. Consider the plane curve $\mathcal{C}: z^{p}-x^{q}=0$. The fundamental group of its complement was determined by Oka $[\mathrm{Ok}]$. A look at Oka's computation reveals that the braid monodromy generator is $\left(\sigma_{1} \cdots \sigma_{p-1}\right)^{q} \in B_{p}$. For instance, to a simple tangency corresponds $\sigma_{1}$, to a node, $\sigma_{1}^{2}$, and to a cusp, $\sigma_{1}^{3}$.

Example 4.6. By the above, the braid monodromy generator of a central line arrangement $\mathcal{A}: z^{n}-x^{n}=0$ is a full twist on $n$ strands, $\Delta^{2}=\left(\sigma_{1} \cdots \sigma_{n-1}\right)^{n} \in B_{n}$ (see also [Hir $]$ ).

Step 2. The conjugating braids $\beta_{k}$ depend on the relative positions of the singularities of $\mathcal{C}$. These braids may be specified as follows. Let $\eta_{k}$ denote the portion of the path $\xi_{k}$ from $y_{0}$ to just before $y_{k}$. The braid $\beta_{k}$ is identified by tracking the components of the fiber of the polynomial cover $\pi=\pi_{f}: \mathcal{C} \backslash \mathcal{C} \cap \mathcal{L} \rightarrow \mathbb{C} \backslash \mathcal{Y}$ of (1) over $\eta_{k}$. Generically, these components have distinct real parts. Braiding occurs when the real parts of two components coincide. We record this braiding by analyzing the imaginary parts of the components, as indicated in the figure below.

More explicitly, recall that the polynomial cover $\pi$ is embedded in the trivial line bundle $\operatorname{pr}_{1}:(\mathbb{C} \backslash \mathcal{Y}) \times \mathbb{C} \rightarrow \mathbb{C} \backslash \mathcal{Y}$. Let $y_{k}^{\prime}=y_{k}+\epsilon$ denote the endpoint of the path $\eta_{k}$. Without loss of generality, we may assume that the components of $\pi^{-1}\left(y_{k}^{\prime}\right)$ (resp. $\pi^{-1}\left(y_{0}\right)$ ) have distinct real parts. After an isotopy of $\mathcal{C}$, we may assume further that the positions of the components of $\pi^{-1}\left(y_{k}^{\prime}\right)$ in $\operatorname{pr}_{1}^{-1}\left(y_{k}^{\prime}\right)=\mathbb{C}$ are identical to those of $\pi^{-1}\left(y_{0}\right)$ in $\operatorname{pr}_{1}^{-1}\left(y_{0}\right)=\mathbb{C}$. Then the image of the path $\eta_{k}$ 
under the coefficient map $a: \mathbb{C} \backslash \mathcal{Y} \rightarrow B^{n}$ is a loop $a\left(\eta_{k}\right)$ in configuration space, and the braid $\beta_{k}$ is the homotopy class of this loop.

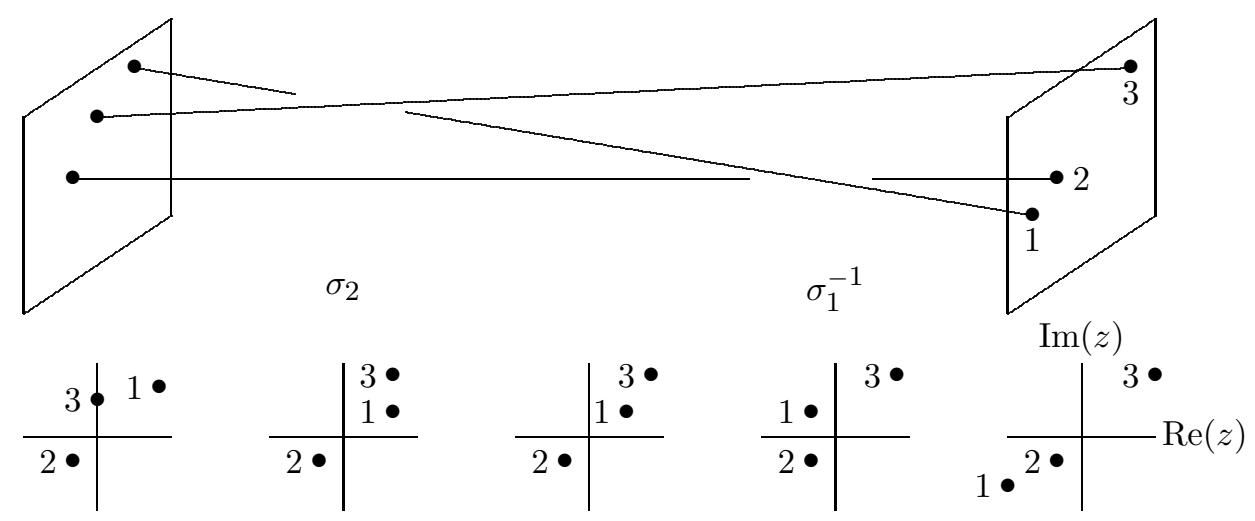

Figure 1. Braiding in a polynomial cover

Remark 4.7. The closed braid determined by the product, $\alpha\left(x_{1}\right) \cdots \alpha\left(x_{s}\right)$, of the braid monodromy generators is the link of the curve $\mathcal{C}$ at infinity. In the works of Moishezon and Libgober, it is usually assumed that $\mathcal{C}$ is in general position relative to the line at infinity in $\mathbb{C P}^{2}$. In that situation, the link at infinity is the $n$-component Hopf link, and thus we have $\alpha\left(x_{1}\right) \cdots \alpha\left(x_{s}\right)=\Delta^{2}$ by Example 4.6.

\section{The braid monodromy of a complex arrangement}

The fundamental group of the complement of an arrangement of complex hyperplanes is, by a well-known Lefschetz-type theorem of Zariski, isomorphic to that of a generic two-dimensional section. So let $\mathcal{A}$ be an arrangement of $n$ complex lines in $\mathbb{C}^{2}$, with group $G=\pi_{1}\left(\mathbb{C}^{2} \backslash \mathcal{A}\right)$. In this section, we provide an explicit description of the pure braid monodromy of $\mathcal{A}$.

\subsection{Braided wiring diagrams}

Choose coordinates in $\mathbb{C}^{2}$ so that the projection $\pi=\operatorname{pr}_{1}: \mathbb{C}^{2} \rightarrow \mathbb{C}$ is generic with respect to $\mathcal{A}$, and let $f(x, z)=\prod_{i=1}^{n}\left(z-\ell_{i}(x)\right)$ be a defining polynomial for $\mathcal{A}$. The points $y_{k} \in \mathcal{Y}$ are the images under $\pi$ of the vertices of $\mathcal{A}$. These vertices, the points $v_{k}=\left(y_{k}, z_{k}\right) \in \mathbb{C}^{2}$ where $z_{k}=\ell_{i_{1}}\left(y_{k}\right)=\cdots=\ell_{i_{r}}\left(y_{k}\right)$ for $r \geq 2$, are the only singularities of $\mathcal{A}$; there are no tangencies. Without loss of generality, assume that the points $y_{k}$ have distinct real parts. As noted in 4.4, Step 1, the local monodromy around $y_{k}$ depends only on $v_{k}$. It is completely determined by the multiplicity of $v_{k}$, and the relative positions of the lines incident on $v_{k}$. 
These data, and the braiding of the lines of $\mathcal{A}$ over the paths $\eta_{k}$, determine the braid monodromy of the arrangement. All of this information may be effectively recorded as follows.

Order the points of $\mathcal{Y}$ as before, and choose the basepoint $y_{0} \in \mathbb{C} \backslash \mathcal{Y}$ so that $\operatorname{Re}\left(y_{0}\right)>\operatorname{Re}\left(y_{1}\right)>\cdots>\operatorname{Re}\left(y_{s}\right)$. Let $\xi:[0,1] \rightarrow \mathbb{C}$ be a (smooth) path emanating from $y_{0}$ and passing through $y_{1}, \ldots, y_{s}$ in order. Note that we may take the path $\xi$ to be a horizontal line segment in a neighborhood of each $y_{k}$. Call such a path admissible. Let

$$
\mathcal{W}=\{(x, z) \in \xi \times \mathbb{C} \mid f(x, z)=0\}
$$

be the braided wiring diagram associated to $\mathcal{A}$. Note that $\mathcal{W}$ depends on the generic linear projection $\pi$ and on the admissible path $\xi$. If $\left\{z=\ell_{i}(x)\right\}$ is a line of $\mathcal{A}$, we call $\mathcal{W} \cap\left\{z=\ell_{i}(x)\right\}$ the associated wire. Since the path $\xi$ passes through the points of $\mathcal{Y}$, the vertices of $\mathcal{A}$ are contained in $\mathcal{W}$.

Over portions of the path $\xi$ between the points of $\mathcal{Y}$, the lines of $\mathcal{A}$ (resp. wires of $\mathcal{W}$ ) may braid. Let $y_{k}^{\prime}=y_{k}+\epsilon$, and $y_{k}^{\prime \prime}=y_{k}-\epsilon$, for some sufficiently small $\epsilon$. We may assume that, over $y_{k}^{\prime}$ and $y_{k}^{\prime \prime}$, the wires of $\mathcal{W}$ (i.e., the components of the fiber of the polynomial cover $\pi_{f}$ ) have distinct real parts. Arguing as in 4.4, Step 2, we associate a braid $\beta_{k, k+1}$ to the portion of $\xi$ from $y_{k}^{\prime \prime}$ to $y_{k+1}^{\prime}$.

After an isotopy of $\mathcal{A}$, we may also assume that the positions of the wires of $\mathcal{W}$ over the points $y_{0}, y_{k}^{\prime}$, and $y_{k}^{\prime \prime}$ are all identical. Thus a braided wiring diagram $\mathcal{W}$ may be abstractly specified by a sequence of states, vertices, and braids:

$$
\Pi_{s+1} \stackrel{\beta_{s, s+1}}{\longleftarrow} V_{s} \Pi_{s} \longleftarrow \leftarrow \cdots \leftarrow \leftarrow \Pi_{2} \longleftarrow \beta_{1,2} \longleftarrow V_{1} \Pi_{1} \stackrel{\beta_{0,1}}{\longleftarrow} \Pi_{0},
$$

where the states $\Pi_{k}$ are permutations of $\{1, \ldots, n\}$ beginning with the identity permutation and recording the relative heights of the wires. The vertex set $V_{k}=$ $\left\{i_{1}, \ldots, i_{r}\right\}$ records the indices of the wires incident on the $k^{\text {th }}$ vertex $v_{k}$ of $\mathcal{A}$ (in terms of the order given by the initial state $\Pi_{0}$ ). The braids $\beta_{k, k+1}$ are obtained as above. By choosing the basepoint $y_{0}$ sufficiently close to $y_{1}$, we may assume that the initial braid $\beta_{0,1}$ is trivial. If such a diagram is depicted as above, the braids $\beta_{k, k+1}$ should be read off from left to right. Note that the this notion generalizes that of a wiring diagram due to Goodman [Go], and that the admissible 2-graphs utilized by Arvola [Ar], [OT], may be viewed as examples of braided wiring diagrams. Explicit examples are given in section 7.

\subsection{Generators of $P_{n}$}

Before proceeding, we need to review some facts about the pure braid group $P_{n}=$ $\operatorname{ker}\left(\tau_{n}: B_{n} \rightarrow \Sigma_{n}\right)$. This group has generators

$$
A_{i, j}=\sigma_{j-1} \cdots \sigma_{i+1} \cdot \sigma_{i}^{2} \cdot \sigma_{i+1}^{-1} \cdots \sigma_{j-1}^{-1}, \quad 1 \leq i<j \leq n,
$$

and relations that set a generator equal to a certain conjugate of itself, see [Bi], [Ha]. In particular, $H_{1}\left(P_{n}\right)=\mathbb{Z}^{\left(\begin{array}{l}n \\ 2\end{array}\right)}$ is generated by the images of the generators 
$A_{i, j}$. The conjugation action of $B_{n}$ on $P_{n}$ is given by the following formulas (compare $[\mathrm{DG}])$ :

$$
A_{i, j}^{\sigma_{k}}=\left\{\begin{array}{ll}
A_{i-1, j} & \text { if } k=i-1, \\
A_{i+1, j}^{A_{i, j+1}} & \text { if } k=i<j-1, \\
A_{i, j-1} & \text { if } k=j-1>i, \\
A_{i, j+1}^{A_{j, j+1}} & \text { if } k=j, \\
A_{i, j} & \text { otherwise }
\end{array} \quad A_{i, j}^{\sigma_{k}^{-1}}= \begin{cases}A_{i-1, j}^{A_{i-1, i}^{-1}} & \text { if } k=i-1, \\
A_{i+1, j} & \text { if } k=i<j-1, \\
A_{i, j-1}^{A_{j-1, j}} & \text { if } k=j-1>i, \\
A_{i, j+1} & \text { if } k=j, \\
A_{i, j} & \text { otherwise. }\end{cases}\right.
$$

We shall work mainly with a particular type of pure braids. These "twist braids" are defined as follows. Given an increasingly ordered set $I=\left\{i_{1}, \ldots, i_{r}\right\}$, let

$$
A_{I}=\left(A_{i_{1}, i_{2}}\right)\left(A_{i_{1}, i_{3}} A_{i_{2}, i_{3}}\right)\left(A_{i_{1}, i_{4}} A_{i_{2}, i_{4}} A_{i_{3}, i_{4}}\right) \cdots\left(A_{i_{1}, i_{r}} \cdots A_{i_{r-1}, i_{r}}\right) .
$$

We extend this definition to sets which are not increasingly ordered (such as the vertex sets $V_{k}$ in 5.1) by first ordering, then proceeding as above. The conjugation action of an arbitrary braid $\beta \in B_{n}$ on the twist braid $A_{I} \in P_{n}$ takes the form

$$
A_{I}^{\beta}=A_{\omega(I)}^{C},
$$

where $\omega=\tau_{n}(\beta)$, and $C=C(I, \beta)$ is a pure braid that may be computed recursively from (5).

\subsection{Braid monodromy}

We now extract the braid monodromy of $\mathcal{A}$ from an associated braided wiring diagram $\mathcal{W}$. By Theorem 3.4, the image of the braid monodromy is contained in the pure braid group $P_{n}$. We shall express the braid monodromy generators, $\lambda_{k}:=\lambda\left(x_{k}\right)$, in terms of the standard generators $A_{i, j}$.

The vertex set $V_{k}=\left\{i_{1}, \ldots, i_{r}\right\}$ gives rise to a partition $\Pi_{k}=L_{k} \cup V_{k} \cup U_{k}$, where $L_{k}$ (resp. $U_{k}$ ) consists of the indices of the wires below (resp. above) the vertex $v_{k}$. Let $I_{k}=\{j, j+1, \ldots, j+r-1\}$ denote the local index of $V_{k}$, where $j=\left|L_{k}\right|+1$. The local monodromy $\gamma_{k}$ around the point $y_{k} \in \mathcal{Y}$ is a full twist on $I_{k}$ given by the pure braid $A_{I_{k}}$ (compare 4.6). Note that $A_{I_{k}}=\mu_{I_{k}}^{2}$, where

$$
\mu_{I_{k}}=\left(\sigma_{j} \cdots \sigma_{j+r-2}\right)\left(\sigma_{j} \cdots \sigma_{j+r-3}\right) \cdots\left(\sigma_{j} \cdot \sigma_{j+1}\right) \cdot\left(\sigma_{j}\right)
$$

is a permutation braid - a half twist on $I_{k}$. Also notice that the monodromy along a path from $y_{k}^{\prime}$ to $y_{k}^{\prime \prime}$ above (or below) the point $y_{k}$ is given by $\mu_{I_{k}}$.

To specify the braid monodromy of $\mathcal{A}$, it remains to identify the conjugating braids $\beta_{k}$ of 4.4, Step 2. Choosing the paths $\eta_{k}$ to coincide with $\xi$ between $y_{j}^{\prime \prime}$ and $y_{j+1}^{\prime}$ for $j<k$, these conjugating braids may be expressed as $\beta_{1}=\beta_{0,1}=1$, and 
$\beta_{k+1}=\beta_{k, k+1} \cdot \mu_{I_{k}} \cdot \beta_{k}$ for $k \geq 1$. Hence the braid monodromy generators are given by

$$
\lambda_{k}=A_{I_{k}}^{\beta_{k}} .
$$

Note that the state $\Pi_{k}$ of the braided wiring diagram $\mathcal{W}$ is the image of the braid $\beta_{k}$ in the symmetric group, $\Pi_{k}=\tau_{n}\left(\beta_{k}\right)$. Note also that the vertex set $V_{k}$ and its local index $I_{k}$ are related by $V_{k}=\Pi_{k}\left(I_{k}\right)$. Thus, the braid monodromy generators may be expressed solely in terms of pure braids:

$$
\lambda_{k}=A_{V_{k}}^{C_{k}},
$$

for certain $C_{k} \in P_{n}$.

\subsection{Conjugate arrangements}

Let $\mathcal{A}$ be an arrangement of $n$ lines in $\mathbb{C}^{2}$, with associated braided wiring diagram $\mathcal{W}$ corresponding to a generic projection $\pi: \mathbb{C}^{2} \rightarrow \mathbb{C}$ and admissible path $\xi$. Let $\overline{\mathcal{A}}$ denote the conjugate arrangement (see 3.8). Clearly, the vertices of $\overline{\mathcal{A}}$ are the complex conjugates of those of $\mathcal{A}$. Thus, $\pi$ is generic with respect to $\overline{\mathcal{A}}$, and $\bar{\xi}$ is admissible. The corresponding braided diagram, $\overline{\mathcal{W}}$, is then obtained from $\mathcal{W}$ by simply reversing the crossings of all the intermediate braids. Thus, the local indices of $\overline{\mathcal{W}}$ are given by $\bar{I}_{k}=I_{k}$, the conjugating braids by $\bar{\beta}_{k+1}=\epsilon_{n}\left(\beta_{k, k+1}\right) \cdot \mu_{I_{k}} \cdot \bar{\beta}_{k}$, and the braid monodromy generators by $\bar{\lambda}_{k}=A_{I_{k}}^{\bar{\beta}_{k}}$. From the proof of Theorem 3.9, we see that the braid monodromy generators of the two conjugate arrangements are related by

$$
\bar{\lambda}_{k}=\epsilon_{n}\left(\left(\lambda_{k}^{-1}\right)^{\lambda_{k-1}^{-1} \cdots \lambda_{1}^{-1}}\right)
$$

\subsection{Real arrangements}

If $\mathcal{A}$ is a real arrangement in $\mathbb{C}^{2}$ (that is, $\mathcal{A}$ is the complexification of a line arrangement $\mathcal{A}_{\mathbb{R}}$ in $\mathbb{R}^{2}$ ), then the defining polynomial $f(x, z)$ has real coefficients. Consequently, the vertices of $\mathcal{A}$ all have real coordinates, and their images under first-coordinate projection all lie on the real axis in $\mathbb{C}$. In this instance, we may take the path $\xi:[0,1] \rightarrow \mathbb{C}$ to be a directed line segment along the real axis. The resulting diagram $\mathcal{W}$ is unbraided - all the intermediate braids $\beta_{k, k+1}$ are trivial. In other words, the diagram is a wiring diagram in the combinatorial sense [Go], affine if $\mathcal{A}$ contains parallel lines (see also [BLSWZ]). In this instance, the description of the braid monodromy given in 5.3 specializes to the algorithm of Hironaka [Hir], modulo some notational differences.

Another description of the braid monodromy of an abstract (unbraided) wiring diagram was provided by Cordovil and Fachada in $[\mathrm{CF}]$ (see also [Cor]). This description, based on Salvetti's work [S1], [S2], may be paraphrased as follows.

Recall that the vertex set $V_{k}=\left\{i_{1}, \ldots, i_{r}\right\}$ gives rise to a partition $\Pi_{k}=$ $L_{k} \cup V_{k} \cup U_{k}$. Let $\bar{V}_{k}=\left\{i \mid i_{1} \leq i \leq i_{r}\right\}$, and set $J_{k}=\left(\bar{V}_{k} \backslash V_{k}\right) \cap U_{k}$. Let 
$B_{J_{k}}=\prod A_{j, i}$, where the product is over all $j \in J_{k}$ and $i \in V_{k}$ with $j<i$, taken in the natural order (so that $B_{J_{k}}$ is a subword of $\Delta^{2}=A_{1, \ldots, n}$, equal to 1 if $J_{k}=\emptyset$ ). Then the braid monodromy generators may be expressed as $\hat{\lambda}_{k}=A_{V_{k}}^{J_{k}}:=B_{J_{k}}^{-1} A_{V_{k}} B_{J_{k}}$, where $A_{V_{k}}$ is as defined in (6).

Using the Artin representation, one can show that the braids $\lambda_{k}$ and $\hat{\lambda}_{k}$ are equal. The action of the braid $\hat{\lambda}_{k}$ is given in formulas (12) and (13) in section 6 . The same formulas hold for $\lambda_{k}$, but the computation is more involved. Thus, the two descriptions of the braid monodromy of a real arrangement (or more generally, of an arbitrary wiring diagram) coincide.

\subsection{Markov moves}

For an arbitrary complex arrangement $\mathcal{A}$, changes in the choices made in the construction of the braid monodromy (see 3.5) give rise to changes in the braided wiring diagram $\mathcal{W}$ associated to $\mathcal{A}$. For instance, changing the basepoint $y_{0}$ may alter the initial braid $\beta_{0,1}$, while changes in the generic projection may alter the order of the vertices.

We refer to these (and other) changes in a braided wiring diagram as "Markov moves." In practice, these moves may be used to simplify a braided wiring diagram associated to an arrangement $\mathcal{A}$ (and consequently the braid monodromy generators of $\mathcal{A}$ as well). We now record these simplifying Markov moves and their effects on the braid monodromy. In the following, we record only the local index of a vertex, so "vertex $\{j, \ldots, k\}$ " means "a vertex with local index $\{j, \ldots, k\}$." Recall that, while we depict braided wiring diagrams right to left, their intermediate braids are read left to right.

\section{Geometric moves.}

(1) Insert an arbitrary braid $\beta_{0}$ at the beginning of the braided wiring diagram.

(2) Insert an arbitrary braid $\beta_{s+1}$ at the end of the braided wiring diagram.

(3) Replace vertex $\{i, \ldots, j\}$, then vertex $\{k, \ldots, l\}$ with

(a) vertex $\{k, \ldots, l\}$, then vertex $\{i, \ldots, j\}$, if $j<k$ or $i>l$.

(b) braid $\left(\sigma_{k} \cdots \sigma_{i+1}\right) \cdot\left(\sigma_{k+1} \cdots \sigma_{i+2}\right) \cdots\left(\sigma_{l-1} \cdots \sigma_{j}\right)$, then vertex $\{i, \ldots, i+l-k\}$, then vertex $\{i+l-k, \ldots, l\}$, then braid $\left(\sigma_{k-1}^{-1} \cdots \sigma_{i}^{-1}\right) \cdot\left(\sigma_{k}^{-1} \cdots \sigma_{i+1}^{-1}\right) \cdots\left(\sigma_{l-2}^{-1} \cdots \sigma_{j-1}^{-1}\right)$, if $j=k$.

(c) braid $\left(\sigma_{i-1} \cdots \sigma_{k}\right) \cdot\left(\sigma_{i} \cdots \sigma_{k+1}\right) \cdots\left(\sigma_{j-2} \cdots \sigma_{l-1}\right)$, then vertex $\{j+k-l, \ldots, j\}$, then vertex $\{k, \ldots, j+k-l\}$, then braid $\left(\sigma_{i}^{-1} \cdots \sigma_{k+1}^{-1}\right) \cdot\left(\sigma_{i+1}^{-1} \cdots \sigma_{k+2}^{-1}\right) \cdots\left(\sigma_{j-1}^{-1} \cdots \sigma_{l}^{-1}\right)$, if $i=l$. 

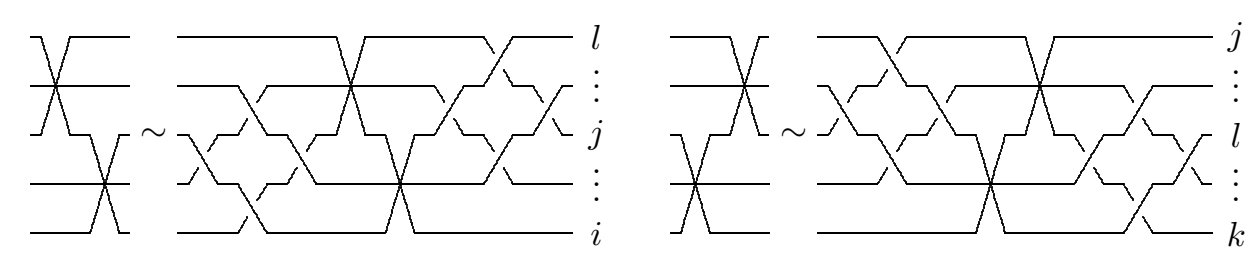

Figure 2. Moves 3b (left) and 3c (right)

\section{Further moves.}

(4) Reduce the intermediate braid $\beta_{k, k+1}$.

(5) Replace braid $\sigma_{i}$, then vertex $\{j, \ldots, k\}$ with

(a) vertex $\{j, \ldots, k\}$, then braid $\sigma_{i}$, if $i<j-1$ or $i>k$.

(b) braid $\sigma_{j}^{-1} \cdots \sigma_{k-1}^{-1}$, then vertex $\{j+1, \ldots, k+1\}$, then braid $\sigma_{k} \cdots \sigma_{j}$, if $i=k$.

(c) braid $\sigma_{k-1}^{-1} \cdots \sigma_{j}^{-1}$, then vertex $\{j-1, \ldots, k-1\}$, then braid $\sigma_{j-1} \cdots \sigma_{k-1}$, if $i=j-1$.

(d) vertex $\{j, \ldots, k\}$, then braid $\sigma_{j+k-i-1}$, if $j \leq i \leq k-1$.



Figure 3. Moves 5b (left), 5c (right), and 5d (bottom)

The parity of the braids in move (3) and move (5) may be switched. For instance, one can replace braid $\sigma_{i}^{-1}$, then vertex $\{j, \ldots, k\}$, with braid $\sigma_{j} \cdots \sigma_{k-1}$, then vertex $\{j+1, \ldots, k+1\}$, then braid $\sigma_{k}^{-1} \cdots \sigma_{j}^{-1}$ if $i=k$ (move $5 \mathrm{~b}$ ).

Note that the triangle-switches and flips discussed in [BLSWZ] and [Fa] in the context of (unbraided) wiring diagrams may be accomplished using Markov moves of types (3), (4), and (5).

Theorem 5.7. The braid monodromy of a braided wiring diagram is invariant under Markov moves: If the braided wiring diagram $\widehat{\mathcal{W}}$ is obtained from the braided wiring diagram $\mathcal{W}$ by a finite sequence of Markov moves of types (1)-(5) and their inverses, then the braid monodromy homomorphisms $\lambda$ of $\mathcal{W}$ and $\hat{\lambda}$ of $\widehat{\mathcal{W}}$ are braid-equivalent.

Sketch of Proof. One can check, either algebraically or by drawing the appropriate 
braids, that the (only) effects on the braid monodromy of the Markov moves listed above are as follows:

(1) Global conjugation by $\beta_{0}: \quad \hat{\lambda}=\beta_{0}^{-1} \cdot \lambda \cdot \beta_{0}$.

(2) None.

(3) Suppose the vertices in question are the $k^{\text {th }}$ and $(k+1)^{\text {st }}$ vertices of $\mathcal{W}$ (resp. $\widehat{\mathcal{W}}$ ). Then the corresponding braid monodromy generators, $\lambda_{k}, \lambda_{k+1}$ and $\hat{\lambda}_{k}, \hat{\lambda}_{k+1}$, satisfy:

(a) $\hat{\lambda}_{k}=\lambda_{k+1}$ and $\hat{\lambda}_{k+1}=\lambda_{k}$. Note that the permutation braids $\mu_{I_{k}}$ and $\mu_{I_{k+1}}$ commute in this instance. Thus we can write $\hat{\lambda}_{k}=\lambda_{k} \cdot \lambda_{k+1} \cdot \lambda_{k}^{-1}=\lambda_{k+1}$, and $\hat{\lambda}=\lambda \circ \sigma_{k}$.

(b) $\hat{\lambda}_{k}=\lambda_{k} \cdot \lambda_{k+1} \cdot \lambda_{k}^{-1}$ and $\hat{\lambda}_{k+1}=\lambda_{k}$. Thus $\hat{\lambda}=\lambda \circ \sigma_{k}$.

(c) $\hat{\lambda}_{k}=\lambda_{k+1}$ and $\hat{\lambda}_{k+1}=\lambda_{k+1}^{-1} \cdot \lambda_{k} \cdot \lambda_{k+1}$. Thus $\hat{\lambda}=\lambda \circ \sigma_{k}^{-1}$.

(4) None.

(5) None.

Hence $\lambda$ and $\hat{\lambda}$ are braid-equivalent.

Recall that curves in the same connected component of an equisingular family have braid-equivalent monodromies (see [L3] and 3.5). In particular, this is the case for arrangements which are lattice-isotopic [R2].

Corollary 5.8. Let $\mathcal{A}$ and $\mathcal{A}^{\prime}$ be lattice-isotopic arrangements in $\mathbb{C}^{2}$, with associated braided wiring diagrams $\mathcal{W}$ and $\mathcal{W}^{\prime}$. Then $\mathcal{W}^{\prime}$ may be obtained from $\mathcal{W}$ via a finite sequence of Markov moves and their inverses.

\section{The group of a complex arrangement}

We now turn our attention the fundamental group $G$ of the complement of the line arrangement $\mathcal{A}$ in $\mathbb{C}^{2}$. In this section, we describe the braid monodromy and Arvola presentations of $G$, show that they are Tietze-I equivalent, and derive some homotopy type consequences.

\subsection{Presentations}

Using the (pure) braid monodromy generators $\left\{\lambda_{k}\right\}$ from (9) and the procedure described in 4.1, we obtain the braid monodromy presentation

$$
\left.G=\left\langle t_{1}, \ldots, t_{n}\right| \lambda_{k}\left(t_{i}\right)=t_{i} \text { for } i \in \check{V}_{k} \text { and } 1 \leq k \leq s\right\rangle,
$$

where $\check{V}_{k}=V_{k} \backslash \max V_{k}$.

We may also use the braided wiring diagram $\mathcal{W}$ to find the Arvola presentation of $G$. This presentation is obtained by applying the Arvola algorithm [Ar], [OT], 
to $\mathcal{W}$. Explicitly, we sweep a vertical line across the braided wiring diagram from right to left, introducing relations and keeping track of conjugations as we pass through the vertices $v_{k}$ and the braids $\beta_{k, k+1}$. Note that the braids $\beta_{k, k+1}$ do not give rise to any relations, but do cause additional conjugations. It is convenient to express the generators and these relations and conjugations in terms of the inverses of the generators typically used in the Arvola algorithm. Apart from this notational difference, our description of this method follows Falk's discussion in $[\mathrm{Fa}]$ of the Randell algorithm for real arrangements (to which the Arvola algorithm specializes).

Recall that the symbol $\left[g_{1}, \ldots, g_{r}\right]$ denotes the family of $r-1$ relations

$$
g_{1} \cdot g_{2} \cdots g_{m}=g_{2} \cdots g_{m} \cdot g_{1}=\cdots \cdots=g_{m} \cdot g_{1} \cdots g_{m-1}
$$

The Arvola presentation of the group $G$ is given by

$$
G=\left\langle t_{1}, \ldots, t_{n} \mid \mathbf{R}_{1}, \ldots, \mathbf{R}_{s}\right\rangle,
$$

where if $V_{k}=\left\{i_{1}, \ldots, i_{r}\right\}$, then $\mathbf{R}_{k}$ denotes the family of relations $\left[x_{i_{1}}(k), \ldots, x_{i_{r}}(k)\right]$. The word $x_{i}(k)$ denotes the meridian about wire $i$ at state $\Pi_{k}$. Let $y_{i}(k)$ denote the meridian about wire $i$ between vertex $k$ and braid $k$. Then we have

$$
y_{i}(k)= \begin{cases}x_{i}(k) & \text { if } i \notin V_{k}, \\ x_{i_{1}}(k) \cdots x_{i_{l}}(k) \cdot x_{i}(k) \cdot\left(x_{i_{1}}(k) \cdots x_{i_{l}}(k)\right)^{-1} & \text { if } i=i_{l+1} \in V_{k} .\end{cases}
$$

(see below). The words $x_{i}(k)$ satisfy the recursion $x_{i}(1)=t_{i}$, and $x_{i}(k+1)=$ $\hat{\beta}_{k, k+1}\left(y_{i}(k)\right)$ for $k>0$, where $\hat{\beta}_{k, k+1}$ records the effect of the braiding $\beta_{k, k+1}$ on the meridian $y_{i}(k)$ as indicated below.
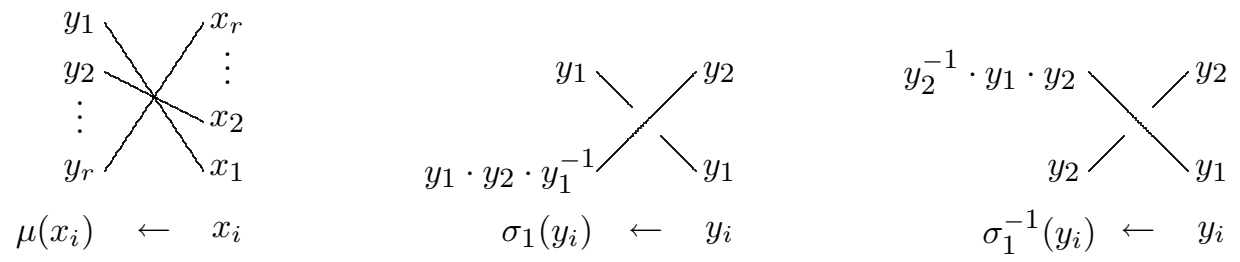

Figure 4. Conjugations in Arvola's algorithm

Note that, locally, the conjugation arising from a vertex (resp. braiding) coincides with the action of a permutation braid (resp. an elementary braid or its inverse). To make the description of $\hat{\beta}_{k, k+1}$ explicit, we require some notation.

The state $\Pi_{k}$ of the braided wiring diagram $\mathcal{W}$ is a permutation of $\{1, \ldots, n\}$, recording the relative heights of the wires at this state. Recall the local index $I_{k}$ of the vertex set $V_{k}$ of $\mathcal{W}$, and the associated permutation braid $\mu_{I_{k}}$. Let $\bar{\mu}_{I_{k}}=$ $\tau_{n}\left(\mu_{I_{k}}\right)$ denote the permutation induced by $\mu_{I_{k}}$, and let $\bar{\beta}_{k, k+1}=\tau_{n}\left(\beta_{k, k+1}\right)$. It is easily seen that $\Pi_{k+1}=\bar{\beta}_{k, k+1} \cdot \bar{\mu}_{I_{k}} \cdot \Pi_{k}$. 
Note that the sets $\left\{x_{i}(k)\right\}$ and $\left\{y_{i}(k)\right\}$ generate the free group $F_{n}=\left\langle t_{1}, \ldots, t_{n}\right\rangle$. Define $\phi_{k}, \psi_{k} \in \operatorname{Aut}\left(F_{n}\right)$ by

$$
\phi_{k}\left(t_{q}\right)=x_{i}(k) \text { if } \Pi_{k}(q)=i \quad \text { and } \quad \psi_{k}\left(t_{q}\right)=x_{i}(k) \text { if } \bar{\mu}_{I_{k}} \cdot \Pi_{k}(q)=i .
$$

If $\Pi_{k+1}(q)=i$, then the effect of the braiding $\beta_{k, k+1}$ on $y_{i}(k)$ may be expressed as $\hat{\beta}_{k, k+1}\left(y_{i}(k)\right)=\beta_{k, k+1} \cdot \psi_{k}\left(t_{q}\right)=\psi_{k}\left(\beta_{k, k+1}\left(t_{q}\right)\right)$.

Lemma 6.2. We have $\psi_{k}=\mu_{I_{k}} \cdot \phi_{k}$.

Proof. Compute:

$\mu_{I_{k}} \cdot \phi_{k}\left(t_{q}\right)= \begin{cases}\phi_{k}\left(t_{q}\right) & \text { if } q \notin I_{k}, \\ \phi_{k}\left(t_{j} \cdots t_{j+l-1} \cdot t_{j+l} \cdot\left(t_{j} \cdots t_{j+l-1}\right)^{-1}\right) & \text { if } q=j+r-1-l \in I_{k} .\end{cases}$

Checking that this agrees with the description of the meridians $y_{i}(k)$ given in (11), we have $\mu_{I_{k}} \cdot \phi_{k}\left(t_{q}\right)=y_{i}(k)=\psi_{k}\left(t_{q}\right)$ for all $q$.

We now show that the meridians $x_{i}(k)$ may be expressed in terms of the conjugating braids $\beta_{k}$ from the braid monodromy constructions of 4.4 and 5.3. Recall that these braids are defined by $\beta_{1}=1$, and $\beta_{k+1}=\beta_{k, k+1} \cdot \mu_{I_{k}} \cdot \beta_{k}$ for $k \geq 1$.

Proposition 6.3. If wire $i$ is at height $q$ at state $\Pi_{k+1}$ in the braided wiring diagram $\mathcal{W}$ (that is, $\left.\Pi_{k+1}(q)=i\right)$, then $x_{i}(k+1)=\beta_{k+1}\left(t_{q}\right)$.

Proof. We use induction on $k$, with the case $k=0$ trivial.

In general, assume $\Pi_{k+1}(q)=i$. We have

$x_{i}(k+1)=\hat{\beta}_{k, k+1}\left(y_{i}(k)\right)=\beta_{k, k+1} \cdot \mu_{I_{k}} \cdot \phi_{k}\left(t_{q}\right)=\beta_{k, k+1} \cdot \mu_{I_{k}} \cdot \beta_{k}\left(t_{q}\right)=\beta_{k+1}\left(t_{q}\right)$,

using the above lemma, the inductive hypothesis to identify $\phi_{k}=\beta_{k}$, and the identification of the braids $\beta_{k}$ from 5.3.

We may now state and prove the main theorem of this section.

Theorem 6.4. The braid monodromy and Arvola presentations of the group $G$ of the arrangement $\mathcal{A}$ are Tietze-I equivalent.

Proof. Let $V=V_{k}=\left\{i_{1}, \ldots, i_{r}\right\}$ denote the $k^{\text {th }}$ vertex of a braided wiring diagram $\mathcal{W}$ associated to $\mathcal{A}$, with local index $I=I_{k}=\{j, \ldots, j+r-1\}$. Write $\beta=\beta_{k}$ and $\alpha=\alpha_{k}=A_{I}^{\beta}$, and recall that $\check{V}=V \backslash \max V$. Using Proposition 6.3, we may express the family $\mathbf{R}_{k}$ of Arvola relations as

$$
\left[\beta\left(t_{j}\right), \ldots, \beta\left(t_{j+r-1}\right)\right]
$$


We will show that these $r-1$ relations and the braid monodromy relations $\alpha\left(t_{i}\right)=$ $t_{i}, i \in \check{V}$ are equivalent. It is easy to see that the $r-1$ Arvola relations above are equivalent to $\beta\left(A_{I}\left(t_{i}\right)\right)=\beta\left(t_{i}\right), i \in \check{I}$.

Using Proposition 6.3 again, we have $\beta\left(t_{i}\right)=x_{i_{p}}(k)$ if $i=j+p-1 \in I$. Consequently, $\beta\left(t_{i}\right)$ is some conjugate of $t_{i_{p}}$, say $\beta\left(t_{i}\right)=w_{p} \cdot t_{i_{p}} \cdot w_{p}^{-1}$. A computation shows that $\alpha\left(t_{i_{p}}\right)=\alpha\left(w_{p}^{-1}\right) \cdot \beta\left(A_{I}\left(t_{i}\right)\right) \cdot \alpha\left(w_{p}\right)$. Therefore the braid monodromy relation $\alpha\left(t_{i_{p}}\right)=t_{i_{p}}$ may be expressed as $\beta\left(A_{I}\left(t_{i}\right)\right)=\alpha\left(w_{p}\right) \cdot t_{i_{p}} \cdot \alpha\left(w_{p}^{-1}\right)$. Now it follows from the relations $\alpha\left(t_{i}\right)=t_{i}$ for $i \in \check{V}$ that $\alpha\left(t_{i}\right)=t_{i}$ for all $i$. Thus $\alpha\left(w_{p}\right)=w_{p}$, and the braid monodromy relation above is clearly equivalent to the corresponding Arvola relation.

Since the braid monodromy and Arvola presentations of the group $G$ of $\mathcal{A}$ are Tietze-I equivalent, the associated 2-complexes are homotopy equivalent. Furthermore, Libgober's theorem [L1] stated in 4.2 provides a homotopy equivalence between the braid monodromy presentation 2-complex and the complement $\mathbb{C}^{2} \backslash \mathcal{A}$. Thus we obtain the following corollary.

Corollary 6.5. The complement of a complex arrangement $\mathcal{A}$ in $\mathbb{C}^{2}$ has the homotopy type of the 2-complex modeled on the Arvola presentation of the group $G$.

Prior to our obtaining this result, Arvola informed us that he had a proof of it. We are not cognizant of the details of that proof.

\subsection{Real arrangements}

If $\mathcal{A}$ is a real arrangement in $\mathbb{C}^{2}$, then, as noted in 5.5 , the braided wiring diagram $\mathcal{W}$ is unbraided, so is a (possibly affine) wiring diagram. In this instance, Arvola's algorithm specializes to that of Randell [R1]. Using Theorem 6.4, we obtain:

Corollary 6.7. The braid monodromy and Randell presentations of the group $G$ of a real arrangement $\mathcal{A}$ in $\mathbb{C}^{2}$ are Tietze-I equivalent.

As above, combining this result with Libgober's theorem yields the following corollary, which constitutes the main result of Falk [Fa].

Corollary 6.8. The complement of a real arrangement $\mathcal{A}$ in $\mathbb{C}^{2}$ has the homotopy type of the 2-complex modeled on the Randell presentation of the group $G$.

The braid monodromy and Randell presentations of $G$ may be obtained immediately from the description of the generators $\hat{\lambda}_{k}=A_{V_{k}}^{J_{k}}$ in terms of pure braids provided by $[\mathrm{CF}]$ and described in 5.5. This is accomplished by finding the action of the braids $\hat{\lambda}_{k}$. For the sake of completeness, we find the action of $\hat{\lambda}_{k}$ on the entire free group $F_{n}$. 
Write $V=V_{k}$ and $J=J_{k}$. If $V=\left\{i_{1}, \ldots, i_{r}\right\}$, let $t_{V}=t_{i_{1}} \cdots t_{i_{r}}$ (set $t_{V}=1$ if $V=\emptyset$ ). For $i \in \bar{V} \backslash V$, let $V^{<i}=\left\{i_{1}, \ldots, i_{q}\right\}$ and $V^{>i}=\left\{i_{q+1}, \ldots, i_{r}\right\}$ if $i_{q}<i<i_{q+1}$. If $J=\emptyset$, a straightforward computation yields

$$
A_{V}\left(t_{i}\right)= \begin{cases}t_{V} \cdot t_{i} \cdot t_{V}^{-1} & \text { if } i \in V, \\ {\left[t_{V<i}, t_{V>i}\right] \cdot t_{i} \cdot\left[t_{V<i}, t_{V>i}\right]^{-1}} & \text { if } i \in \bar{V} \backslash V \\ t_{i} & \text { otherwise. }\end{cases}
$$

If $J \neq \emptyset$, let

$$
z_{J, i}= \begin{cases}1 & \text { if } i<j_{1} \text { or } i \in J \text { or } i>i_{r} \\ t_{J<i} & \text { if } i \in \bar{J} \backslash J \\ t_{J} & \text { if } \max J<i \leq i_{r}\end{cases}
$$

and define $\gamma_{J} \in \operatorname{Aut}\left(F_{n}\right)$ by $\gamma_{J}\left(t_{i}\right)=z_{J, i} \cdot t_{i} \cdot z_{J, i}^{-1}$. Induction on $|J|$, starting from (12), yields:

$$
A_{V}^{J}\left(t_{i}\right)= \begin{cases}z_{J, i}^{-1} \cdot \gamma_{J}\left(t_{V} \cdot t_{i} \cdot t_{V}^{-1}\right) \cdot z_{J, i} & \text { if } i \in V, \\ z_{J, i}^{-1} \cdot \gamma_{J}\left(\left[t_{V<i}, t_{V>i}\right] \cdot t_{i} \cdot\left[t_{V<i}, t_{V>i}\right]^{-1}\right) \cdot z_{J, i} & \text { if } i \in \bar{V} \backslash(V \cup J), \\ t_{i} & \text { if } i \in J \text { or } i \notin \bar{V} .\end{cases}
$$

Proposition 6.9. Let $\mathcal{A}$ be a real arrangement. If $\mathcal{W}$ is an associated wiring diagram with vertex sets $V_{k}$ and conjugating sets $J_{k}$, then the braid monodromy and Randell presentations of the group $G(\mathcal{A})$ are given by

$$
\begin{aligned}
G & \left.=\left\langle t_{1}, \ldots, t_{n}\right| \gamma_{k}\left(t_{V_{k}} \cdot t_{i} \cdot t_{V_{k}}^{-1}\right)=\gamma_{k}\left(t_{i}\right) \text { for } i \in \check{V}_{k} \text { and } 1 \leq k \leq s\right\rangle \\
& =\left\langle t_{1}, \ldots, t_{n} \mid \mathbf{R}_{1}, \ldots, \mathbf{R}_{s}\right\rangle,
\end{aligned}
$$

where $\gamma_{k}=\gamma_{J_{k}}$, and $\mathbf{R}_{k}$ denotes the family of relations $\left[\gamma_{k}\left(t_{i_{1}}\right), \ldots, \gamma_{k}\left(t_{i_{r}}\right)\right]$.

\section{Applications and examples}

In this section, we demonstrate the techniques described above by means of several explicit examples and provide some applications.

\subsection{The intersection lattice}

Let $\mathcal{A}=\left\{H_{1}, \ldots, H_{n}\right\}$ be an arrangement, and let $L(\mathcal{A})$ be the ranked poset of non-empty intersections of $\mathcal{A}$, ordered by reverse inclusion, and with rank function given by codimension. Two arrangements $\mathcal{A}$ and $\mathcal{A}^{\prime}$ are lattice-isomorphic if there is an order-preserving bijection $\pi: L(\mathcal{A}) \rightarrow L\left(\mathcal{A}^{\prime}\right)$ (see [OT] for further details). 
Let $\mathcal{A}$ be an arrangement of $n$ lines in $\mathbb{C}^{2}$ with $s$ vertices. Choose (arbitrary) orderings of the lines and vertices of $\mathcal{A}$. Then the intersection lattice may be encoded simply by a map $V:\{1, \ldots, s\} \rightarrow \mathcal{S}(n)$, where $\mathcal{S}(n)$ denotes the set of all subsets of $\{1, \ldots, n\}$, and $V(k)=V_{k}$ is the $k^{\text {th }}$ vertex set. Two arrangements $\mathcal{A}$ and $\mathcal{A}^{\prime}$ in $\mathbb{C}^{2}$ are lattice-isomorphic if, upon ordering their respective lines and vertices, there exist permutations $\pi \in \Sigma_{s}$ and $\rho \in \Sigma_{n}$ such that $V_{\pi(k)}^{\prime}=\rho\left(V_{k}\right)$.

Theorem 7.2. Line arrangements with braid-equivalent monodromies are latticeisomorphic.

Proof. First recall that, if $A_{I}$ is one of the (extended) generators of $P_{n}$ specified in (6), and $\beta \in B_{n}$ with $\tau_{n}(\beta)=\omega$, then $A_{I}^{\beta}=A_{\omega(I)}^{C}$, for some $C \in P_{n}$, see (7). Also note that, since the abelianization of $P_{n}$ is a free abelian group on the images of the standard generators $A_{i, j}$, if a pure braid $\gamma$ can be written as $\gamma=B^{-1} \cdot A_{I}^{ \pm 1} \cdot B$, for some $B \in P_{n}$, then the indexing set $I$ is uniquely determined by $\gamma$.

Now let $\mathcal{A}$ and $\mathcal{A}^{\prime}$ be line arrangements with braid-equivalent monodromies $\alpha$ and $\alpha^{\prime}$. Note that the braid monodromy construction determines orderings of the lines and vertices of the arrangements. Write $\alpha\left(x_{k}\right)=A_{V_{k}}^{C_{k}}$ and $\alpha^{\prime}\left(x_{k}\right)=A_{V_{k}^{\prime}}^{C_{k}^{\prime}}$, with $C_{k}, C_{k}^{\prime} \in P_{n}$ as in (10). By assumption, $\alpha^{\prime} \circ \psi=\operatorname{conj}_{\phi} \circ \alpha$, with $\psi \in B_{s}$, $\phi \in B_{n}$. Write $\psi\left(x_{k}\right)=z_{k}^{-1} \cdot x_{\pi(k)} \cdot z_{k}$, where $\pi=\tau_{s}(\psi)$. Also, set $\rho=\tau_{n}(\phi)$. The braid-equivalence then reads:

$$
A_{V_{\pi(k)}^{\prime}}^{C_{k}^{\prime} \alpha^{\prime}\left(z_{k}\right)}=A_{\rho\left(V_{k}\right)}^{B_{k} C_{k}^{\phi}}
$$

Since both exponents in the above equation are pure braids, we conclude that $V_{\pi(k)}^{\prime}=\rho\left(V_{k}\right)$, as required.

Example 7.3. One can easily find pairs of arrangements whose groups are isomorphic, yet whose monodromies are not equivalent. For instance, consider arrangements with defining polynomials $Q(\mathcal{A})=x y(x-y)$ and $Q\left(\mathcal{A}^{\prime}\right)=x y(x-1)$, respectively. It is readily checked that $G(\mathcal{A})=P_{3}$ is isomorphic to $G\left(\mathcal{A}^{\prime}\right)=F_{2} \times F_{1}$. Furthermore, it can be seen that $\mathbb{C}^{2} \backslash \mathcal{A}=\left(S^{3} \backslash 3\right.$ Hopf circles $) \times \mathbb{R}^{+}$is diffeomorphic to $\mathbb{C}^{2} \backslash \mathcal{A}^{\prime}=S^{1} \times\left(S^{2} \backslash 3\right.$ points $) \times \mathbb{R}^{+}$. On the other hand, the respective pure braid monodromies, $\lambda: F_{1} \rightarrow P_{3}, \lambda(x)=A_{1,2,3}$, and $\lambda^{\prime}: F_{2} \rightarrow P_{3}, \lambda^{\prime}\left(x_{1}\right)=A_{1,2}$, $\lambda^{\prime}\left(x_{2}\right)=A_{1,3}$, are obviously not equivalent. This may be explained by the fact that there is no ambient diffeomorphism of $\mathbb{C}^{2}$ taking $\mathcal{A}$ to $\mathcal{A}^{\prime}$.

While these examples do show that the braid monodromy of a plane curve carries more information than the fundamental group of the complement, they are unsatisfying for several reasons. Combinatorially, $L(\mathcal{A})$ is a lattice, while $L\left(\mathcal{A}^{\prime}\right)$ is merely a poset. Geometrically, $\mathcal{A}$ is transverse to the line at infinity, while $\mathcal{A}^{\prime}$ is not. This being the case, these examples do not address Libgober's question [L3], which was posed for plane curves that are transverse to the line at infinity in $\mathbb{C P}^{2}$. We now present examples which do fit into this framework. 


\subsection{Falk arrangements}

Consider the arrangements of Falk $[\mathrm{Fa}]$ defined by

$Q=y z(x+y)(x-y)(x+z)(x-z) \quad$ and $\quad Q^{\prime}=y z(x+z)(x-z)(y-z)(x-y-z)$.

Taking generic sections, we get a pair of real line arrangements $\mathcal{A}$ and $\mathcal{A}^{\prime}$ in $\mathbb{C}^{2}$ which are transverse to the line at infinity, and have the same numbers of double and triple points. Wiring diagrams for these line arrangements are depicted below.
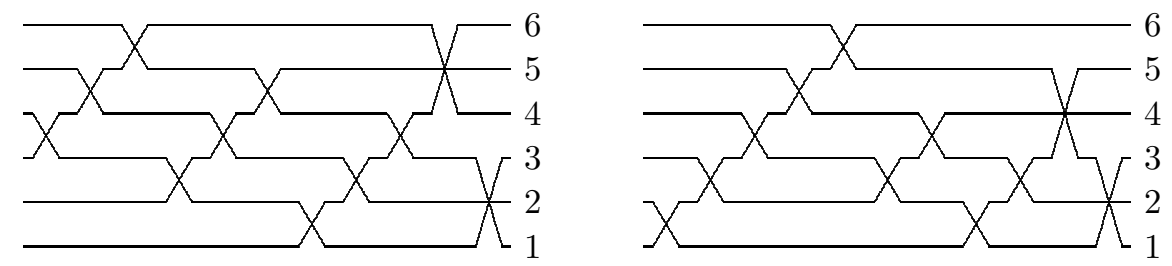

Figure 5. Wiring diagrams for $\mathcal{A}$ (left) and $\mathcal{A}^{\prime}$ (right)

Applying the methods described in the previous sections, we obtain the following braid monodromy generators $\vec{\lambda}=\left\{\lambda_{k}\right\}$ and $\vec{\lambda}^{\prime}=\left\{\lambda_{k}^{\prime}\right\}$ :

$$
\begin{aligned}
\vec{\lambda} & =\left\{A_{1,2,3}, A_{4,5,6}, A_{1,6}^{\{4,5\}}, A_{2,6}^{\{4,5\}}, A_{3,6}^{\{4,5\}}, A_{1,5}^{\{4\}}, A_{2,5}^{\{4\}}, A_{3,5}^{\{4\}}, A_{1,4}, A_{2,4}, A_{3,4}\right\}, \\
\vec{\lambda}^{\prime} & =\left\{A_{1,2,3}, A_{1,4,5}, A_{2,5}^{\{4\}}, A_{3,5}^{\{4\}}, A_{2,4}, A_{3,4}, A_{1,6}, A_{2,6}, A_{3,6}, A_{4,6}, A_{5,6}\right\} .
\end{aligned}
$$

Using Proposition 6.9 and some elementary simplifications, we obtain the following presentations for the groups $G(\mathcal{A})$ and $G\left(\mathcal{A}^{\prime}\right)$ :

$$
\begin{gathered}
G(\mathcal{A})=\left\langle u_{1}, \ldots, u_{6}\right|\left[u_{1}, u_{2}, u_{3}\right],\left[u_{4}, u_{5}, u_{6}\right],\left[u_{1}, u_{6}\right],\left[u_{2}, u_{6}\right],\left[u_{3}, u_{6}\right],\left[u_{1}, u_{5}\right], \\
\left.\left[u_{2}, u_{5}\right],\left[u_{3}, u_{5}\right],\left[u_{1}, u_{4}\right],\left[u_{2}, u_{4}\right],\left[u_{3}, u_{4}\right]\right\rangle, \\
G\left(\mathcal{A}^{\prime}\right)=\left\langle v_{1}, \ldots, v_{6}\right|\left[v_{1}, v_{2}, v_{3}\right],\left[v_{1}, v_{4}, v_{5}\right],\left[v_{2}, v_{5}\right],\left[v_{3}, v_{5}\right],\left[v_{2}, v_{4}\right],\left[v_{3}, v_{4}\right], \\
\left.\left[v_{1}, v_{6}\right],\left[v_{2}, v_{6}\right],\left[v_{3}, v_{6}\right],\left[v_{4}, v_{6}\right],\left[v_{5}, v_{6}\right]\right\rangle .
\end{gathered}
$$

These groups are isomorphic. In fact, one can check that the map $G\left(\mathcal{A}^{\prime}\right) \rightarrow G(\mathcal{A})$ defined by $v_{1} \mapsto u_{1} u_{5}^{-1} u_{4}^{-1}, v_{6} \mapsto u_{4} u_{5} u_{6}, v_{i} \mapsto u_{i}$ if $i \neq 1,6$ is an isomorphism through Tietze-I moves, so the complements of $\mathcal{A}$ and $\mathcal{A}^{\prime}$ are homotopy equivalent. Falk obtained analogous results for the original central 3-arrangements by working with decones as opposed to generic sections.

On the other hand, the lattices of $\mathcal{A}$ and $\mathcal{A}^{\prime}$ are not isomorphic: For $\mathcal{A}^{\prime}$, the two triple points are incident on a line, while for $\mathcal{A}$, they are not. By Theorem 7.2, the monodromies $\lambda, \lambda^{\prime}: F_{11} \rightarrow B_{6}$ are not braid-equivalent. Moreover, the fact that $L(\mathcal{A}) \nRightarrow L\left(\mathcal{A}^{\prime}\right)$ implies, by results of Jiang and Yau [JY], that the complements of $\mathcal{A}$ and $\mathcal{A}^{\prime}$ are not homeomorphic. 


\subsection{Falk-Sturmfels arrangements}

Consider the pair of (central) plane arrangements in $\mathbb{C}^{3}$, with defining polynomials

$$
Q^{ \pm}=x y z(x+y+z)(x+\gamma y)(y+z)(x+(\gamma+1) y+z)(-x+\gamma z)\left(x+\gamma y+\gamma^{2} z\right),
$$

where $\gamma=(-1 \pm \sqrt{5}) / 2$. These arrangements, studied by Falk and Sturmfels (unpublished), are real realizations of a minimal matroid, whose realization space is disconnected (see also [BLSWZ]). They have isomorphic lattices and homotopy equivalent complements. In fact, Keaty (also unpublished) has shown that the oriented matroids of these arrangements are isomorphic. Thus, by results of Björner and Ziegler [BZ], their complements are homeomorphic. Checking that $H^{+}=\left\{z=1-\frac{2}{5} x+\frac{2}{7} y\right\}$ and $H^{-}=\left\{z=1-\frac{4}{9} x+\frac{1}{7} y\right\}$ are generic with respect to these arrangements, we get a pair of real line arrangements, $\mathcal{A}^{ \pm}$, in $\mathbb{C}^{2}$ by taking sections. Wiring diagrams for these line arrangements are depicted below.
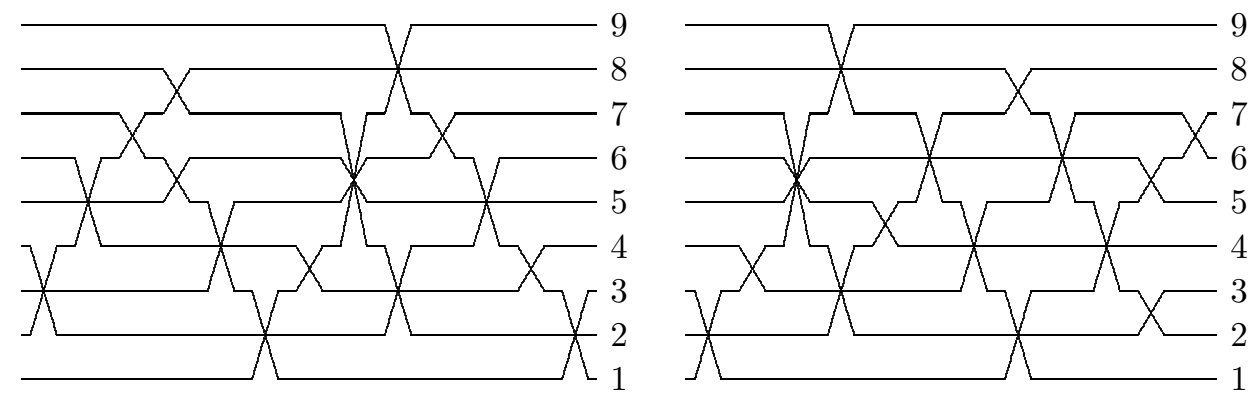

Figure 6. Wiring diagrams for $\mathcal{A}^{+}$(left) and $\mathcal{A}^{-}$(right)

Applying the techniques described in the previous sections, we obtain the following braid monodromy generators:

$$
\begin{gathered}
\vec{\lambda}^{+}=\left\{A_{1,2,3}, A_{1,4}, A_{1,5,6}, A_{1,7}, A_{1,8,9}, A_{2,4,6}^{\{5\}}, A_{2,5,7,9}^{\{8\}}, A_{4,9}^{\{5,7,8\}}, A_{3,6,9}^{\{4,5,7,8\}},\right. \\
\left.A_{3,4,7}^{\{5\}}, A_{2,8}, A_{3,5}, A_{3,8}, A_{4,5,8}, A_{6,7,8}\right\} \\
\vec{\lambda}^{-}=\left\{A_{6,7}, A_{5,7}^{\{6\}}, A_{2,3}, A_{2,4,7}^{\{5,6\}}, A_{2,5,6}, A_{2,8}, A_{1,3,7}^{\{2,4,5,6\}}, A_{1,4,6}^{\{2,5\}}, A_{1,5,8}^{\{2\}},\right. \\
\left.A_{4,8}^{\{5\}}, A_{1,2,9}, A_{3,6,8}^{\{4,5\}}, A_{3,4,5,9}, A_{6,9}, A_{7,8,9}\right\} .
\end{gathered}
$$

The monodromy homomorphisms $\lambda^{+}, \lambda^{-}: F_{15} \rightarrow B_{9}$ are braid-equivalent. If $I=\{i, i+1, \ldots, j\}$, write $\mu_{i, j}=\mu_{I}$, see (8). One can check that $\lambda^{+} \circ \psi=$ $\operatorname{conj}_{\phi} \circ \lambda^{-}$, where $\psi \in B_{15}$ is given by

$$
\psi=\left(\sigma_{1} \sigma_{2} \cdots \sigma_{14}\right)^{4} \sigma_{6} \mu_{3,6} \sigma_{6} \sigma_{10} \sigma_{11} \sigma_{12} \sigma_{13} \mu_{7,10} \sigma_{10} \mu_{11,15} \sigma_{4} \sigma_{5} \sigma_{6} \sigma_{9} \sigma_{10} \mu_{6,9} \sigma_{5} \sigma_{11}
$$


and $\phi \in B_{9}$ is given by $\phi=\left(\sigma_{8} \sigma_{7} \cdots \sigma_{1}\right)^{4} \sigma_{3} \sigma_{4} \sigma_{3} \sigma_{2} \sigma_{5} \sigma_{6}$. It follows from Remark 4.3 that the groups $G\left(\mathcal{A}^{+}\right)$and $G\left(\mathcal{A}^{-}\right)$are isomorphic.

Remark 7.6. If $\lambda: F_{s} \rightarrow P_{n}$ is the braid monodromy of an arrangement or wiring diagram, let $\Gamma=\operatorname{im}(\lambda)<P_{n}$. In $[\mathrm{CF}]$, it is asserted that if $\mathcal{W}$ and $\mathcal{W}^{\prime}$ are wiring diagrams determining the same underlying matroid, then the corresponding braid monodromy subgroups $\Gamma$ and $\Gamma^{\prime}$ of $P_{n}$ are equal. A subsequent result for real arrangements may be found in [Cor].

These results cannot be strengthened. By construction, the wiring diagrams $\mathcal{W}^{+}$and $\mathcal{W}^{-}$above determine isomorphic underlying matroids. However, their braid monodromy groups $\Gamma^{+}$and $\Gamma^{-}$do not coincide. In fact, $\Gamma^{+}$and $\Gamma^{-}$are not conjugate in $P_{9}$ (although, as shown previously, they are conjugate in $B_{9}$ ). This can be seen by using the representation $\theta: P_{9} \rightarrow \operatorname{GL}\left(8, \mathbb{Z}^{10}\right)$, which is obtained from the generalized Gassner representation $\hat{\theta}_{9,2,2}^{1}$ of [CS1], Section 5.8, by restriction to a direct summand. The corresponding modules of coinvariants, $A_{\theta}\left(\mathcal{A}^{ \pm}\right)=H_{0}\left(F_{15} ;\left(\mathbb{Z} \mathbb{Z}^{10}\right)_{\theta \circ \lambda^{ \pm}}^{8}\right)$, are not isomorphic. One can show that the graded modules associated to their $I$-adic completions have different Hilbert series.

This difference may be explained combinatorially as follows. Though the (little) oriented matroids of $\mathcal{W}^{+}$and $\mathcal{W}^{-}$are isomorphic, their big oriented matroids are not. One can check that the spectra of the tope graphs (see [BSLWZ]) associated to these big oriented matroids differ.

\subsection{MacLane configurations}

We consider complex conjugate arrangements arising from the MacLane matroid $\mathrm{ML}_{8}[\mathrm{MacL}]$. This matroid is minimal non-orientable in the sense that it is the smallest matroid that is realizable over $\mathbb{C}$ but not over $\mathbb{R}$ [BLSWZ]. Following Rybnikov [Ry], we take arrangements with defining polynomials

$$
Q^{ \pm}=x y z(y-x)(z-x)(z+\omega y)\left(z+\omega^{2} x+\omega y\right)\left(z-x-\omega^{2} y\right)
$$

where $\omega=(-1 \pm \sqrt{-3}) / 2$, as complex realizations of this matroid. Deconing by setting $x=1$, we obtain two affine arrangements $\mathcal{A}^{ \pm}$in $\mathbb{C}^{2}$. By construction, $\mathcal{A}^{+}$ and $\mathcal{A}^{-}$are lattice-isomorphic. Also, note that $\mathcal{A}^{+}$and $\mathcal{A}^{-}$are conjugate arrangements; in particular, they have diffeomorphic complements, and thus isomorphic groups.

Check that the projection $\pi(y, z)=3 y+z$ is generic with respect to $\mathcal{A}^{+}$. Changing coordinates accordingly, and choosing an admissible path $\xi$, we obtain the braided wiring diagram $\mathcal{W}^{+}$depicted below. 


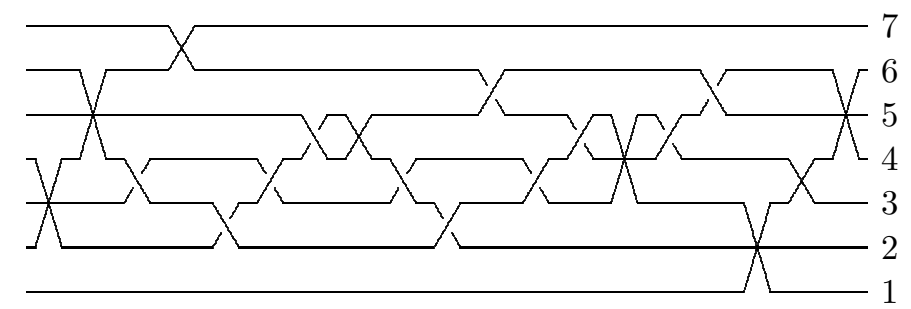

Figure 7. Braided wiring diagram for $\mathcal{A}^{+}$

From the braided wiring diagram $\mathcal{W}^{+}$, we see that

$$
\begin{aligned}
& I_{1}=\{4,5,6\}, \quad \beta_{1,2}=1, \quad I_{2}=\{3,4\}, \quad \beta_{2,3}=1, \\
& I_{3}=\{1,2,3\}, \quad \beta_{3,4}=\sigma_{4} \sigma_{5}^{-1}, \quad I_{4}=\{3,4,5\}, \quad \beta_{4,5}=\sigma_{3}^{-1} \sigma_{2} \sigma_{5} \sigma_{3} \sigma_{4}, \\
& I_{5}=\{4,5\}, \quad \beta_{5,6}=\sigma_{2}^{-1} \sigma_{3} \sigma_{4}^{-1}, \quad I_{6}=\{6,7\}, \quad \beta_{6,7}=\sigma_{3}^{-1}, \\
& I_{7}=\{4,5,6\}, \quad \beta_{7,8}=1, \quad I_{8}=\{2,3,4\} .
\end{aligned}
$$

Since $\mathcal{A}^{+}$and $\mathcal{A}^{-}$are conjugate, a braided wiring diagram $\mathcal{W}^{-}$for $\mathcal{A}^{-}$may be obtained from $\mathcal{W}^{+}$by switching the crossings of the intermediate braids, as noted in 5.4. Applying the algorithm of 5.3 and carrying out some elementary simplifications using (5), (7), and the braid relations, we get the following braid monodromy generators:

$$
\begin{aligned}
& \vec{\lambda}^{+}=\left\{A_{4,5,6}, A_{3,6}, A_{1,2,6}, A_{1,3,4}, A_{2,5}^{A_{3,5} A_{4,5} A_{5,7}}, A_{4,7}, A_{1,5,7}, A_{2,3,7}^{A_{4,7} A_{5,7} A_{3,4}}\right\}, \\
& \vec{\lambda}^{-}=\left\{A_{4,5,6}, A_{3,6}, A_{1,2,6}, A_{1,3,4}, A_{2,5}, A_{4,7}^{A_{5,7}}, A_{1,5,7}, A_{2,3,7}^{A_{5,7}}\right\} .
\end{aligned}
$$

The braid monodromy presentations of the groups $G^{ \pm}=G\left(\mathcal{A}^{ \pm}\right)$may then be found using the Artin representation. After some simplifications, we obtain:

$$
\begin{gathered}
G^{+}=\left\langle u_{1}, \ldots, u_{7}\right|\left[u_{4}, u_{5}, u_{6}\right],\left[u_{3}, u_{6}\right],\left[u_{1}, u_{2}, u_{6}\right],\left[u_{1}, u_{3}, u_{4}\right],\left[u_{2}^{u_{3}}, u_{5}\right], \\
\left.\left[u_{4}, u_{7}\right],\left[u_{1}, u_{5}, u_{7}\right],\left[u_{2}^{u_{6}}, u_{3}, u_{7}\right]\right\rangle, \\
G^{-}=\left\langle v_{1}, \ldots, v_{7}\right|\left[v_{4}, v_{5}, v_{6}\right],\left[v_{3}, v_{6}\right],\left[v_{1}, v_{2}, v_{6}\right],\left[v_{1}, v_{3}, v_{4}\right],\left[v_{2}, v_{5}\right], \\
\left.\left[v_{4}^{v_{5}}, v_{7}\right],\left[v_{1}, v_{5}, v_{7}\right],\left[v_{2}, v_{3}^{v_{5}}, v_{7}\right]\right\rangle .
\end{gathered}
$$

As mentioned above, $G^{+} \cong G^{-}$. An explicit isomorphism is given by

$$
\begin{array}{llll}
u_{1} \mapsto v_{4} v_{1}^{-1} v_{4}^{-1}, & u_{2} \mapsto\left(v_{6} v_{3}\right)^{-1} v_{2}^{-1} v_{6} v_{3}, & u_{3} \mapsto v_{3}^{-1}, & u_{4} \mapsto v_{4}^{-1}, \\
u_{5} \mapsto v_{4} v_{5}^{-1} v_{4}^{-1}, & u_{6} \mapsto v_{6}^{-1}, & u_{7}^{-1} v_{5}^{-1} . &
\end{array}
$$

Presentations for $G^{ \pm}$were first obtained by Rybnikov [Ry], using Arvola's algorithm. By Theorem 6.4, the above presentations are Tietze-I equivalent to those of Rybnikov. This can also be seen directly: For $G^{+}$, an isomorphism is given 
by $u_{1} \mapsto w_{7}^{-1}, u_{2} \mapsto w_{7} w_{4}^{-1} w_{7}^{-1}, u_{3} \mapsto w_{6}^{-1}, u_{4} \mapsto w_{3}^{-1}, u_{5} \mapsto w_{5}^{-1}, u_{6} \mapsto w_{1}^{-1}$, $u_{7} \mapsto w_{2}^{-1}$, and similarly for $G^{-}$.

Since $\mathcal{A}^{+}$and $\mathcal{A}^{-}$are conjugate, their braid monodromies are equivalent by Theorem 3.9. But the two monodromies are not braid-equivalent. For, if they were, there would be an isomorphism $\bar{\phi}: G^{+} \rightarrow G^{-}$determined by a braid automorphism $\phi: F_{7} \rightarrow F_{7}$ (see Remark 4.3). In particular, the induced map on homology, $\bar{\phi}_{*}: H_{1}\left(G^{+}\right) \rightarrow H_{1}\left(G^{-}\right)$, would be a permutation matrix in $\operatorname{GL}(7, \mathbb{Z})$, and this is ruled out by a result of Rybnikov [Ry], Theorem 3.1.

\section{References}

[A-B] E. Artal-Bartolo, Sur les couples de Zariski, J. Alg. Geom. 3 (1994), 223-247.

[Ar] W. Arvola, The fundamental group of the complement of an arrangement of complex hyperplanes, Topology 31 (1992), 757-766.

[Bi] J. Birman, Braids, Links and Mapping Class Groups, Annals of Math. Studies 82, Princeton Univ. Press, 1975.

[BZ] A. Björner, G. Ziegler, Combinatorial stratification of complex arrangements, J. Amer. Math. Soc. 5 (1992), 105-149.

[BLSWZ] A. Björner, M. Las Vergnas, B. Sturmfels, N. White, G. Ziegler, Oriented Matroids, Encyclopedia Math. and Appl. 46, Cambridge Univ. Press, 1993.

[BK] E. Brieskorn, H. Knörrer, Plane Algebraic Curves, Birkhäuser, 1986.

[CS1] D. Cohen, A. Suciu, Homology of iterated semidirect products of free groups, J. Pure Appl. Algebra 126 (1998).

[CS2] D. Cohen, A. Suciu, Alexander invariants of complex hyperplane arrangements, preprint, 1997.

[Cor] R. Cordovil, Braid monodromy groups of arrangements of hyperplanes preprint. 1994.

[CF] R. Cordovil, J. Fachada, Braid monodromy groups of wiring diagrams, Boll. Un. Mat. Ital. 9 (1995), 399-416.

[Di] A. Dimca, Singularities and Topology of Hypersurfaces, Universitext, Springer-Verlag, 1992.

[DH] N. Dung, H. Ha, The fundamental group of complex hyperplane arrangements, Acta Math. Vietnam 20 (1995), 31-41.

[DG] J. Dyer, E. Grossman, The automorphisms groups of the braid groups, Amer. Math. J. 103 (1981), 1151-1169.

[EN] D. Eisenbud, W. Neumann, Three-Dimensional Link Theory and Invariants of Plane Curve Singularities, Annals of Math. Studies 110, Princeton Univ. Press, 1985.

[FN] E. Fadell, L. Neuwirth, Configuration spaces Math. Scand. 10 (1962), 111-118.

[Fa] M. Falk, Homotopy types of line arrangements Invent. Math. 111 (1993), 139-150.

[Go] J. Goodman, Proof of a conjecture of Burr, Grünbaum and Sloane, Discrete Math. 32 (1980), 27-35.

[H1] V. L. Hansen, Coverings defined by Weierstrass polynomials, J. reine angew. Math. 314 (1980), 29-39.

[H2] V. L. Hansen, Braids and Coverings, London Math. Soc. Student Texts 18, Cambridge Univ. Press, 1989.

[Hir] E. Hironaka, Abelian Coverings of the Complex Projective Plane Branched along Configurations of Real Lines, Memoirs AMS 502, Amer. Math. Soc. 1993.

[JY] T. Jiang, S. S.-T. Yau, Topological invariance of intersection lattices of arrangements in $\mathbb{C P}^{2}$, Bull. Amer. Math. Soc. 29 (1993), 88-93. 
[vK ] E. R. van Kampen On the fundamental group of an algebraic plane curve, Amer. J. Math. 55 (1933), 255-260.

[L1 ] A. Libgober, On the homotopy type of the complement to plane algebraic curves, $J$. reine angew. Math. 397 (1986), 103-114.

[L2] A. Libgober, Fundamental groups of the complements to plane singular curves, Proc. Symp. Pure Math. 46 (1987), 29-45.

[L3] A. Libgober, Invariants of plane algebraic curves via representations of the braid groups, Invent. Math. 95 (1989), 25-30.

[MacL] S. MacLane, Some interpretations of abstract linear independence in terms of projective geometry, Amer. J. Math. 58 (1936), 236-241.

[Mo] B. Moishezon, Stable branch curves and braid monodromies, In: Algebraic Geometry., Lect. Notes in Math. 862 Springer-Verlag, 1981, 107-192.

[MT] B. Moishezon, M. Teicher, Braid group technique in complex geometry I: Line arrangements in $\mathbb{C P}^{2}$, In: Braids. Contemporary Math. 78 Amer. Math. Soc, 1988, $425-555$.

[Ok] M. Oka, On the fundamental group of the complement of certain plane curves, $J$. Math. Soc. Japan 30 (1978) 579-597.

[OT ] P. Orlik, H. Terao, Arrangements of Hyperplanes, Grundlehren 300 Springer-Verlag, 1992.

[R1] R. Randell, The fundamental group of the complement of a union of complex hyperplanes, Invent. Math. 69 (1982), 103-108. Correction, Invent. Math. 80 (1985), 467-468.

[R2] R. Randell, Lattice-isotopic arrangements are topologically isomorphic, Proc. Amer. Math. Soc. 107 (1989), 555-559.

[Ru] L. Rudolph, Some knot theory of complex plane curves, In: Nœuds, Tresses et Singularités, L'Enseignement Math. 31, Kundig, 1983, 99-122.

[Ry] G. Rybnikov, On the fundamental group of the complement of a complex hyperplane arrangement, preprint, 1993.

[S1] M. Salvetti, Topology of the complement of real hyperplanes in $\mathbb{C}^{N}$, Invent. Math. 88 (1987), 603-618.

[S2] M. Salvetti, Arrangements of lines and monodromy of plane curves, Compositio Math. 68 (1988), 103-122.

[Z1] O. Zariski, On the problem of existence of algebraic functions of two variables possesing a given branch curve, Amer. J. Math. 51 (1929), 305-328.

[Z2] O. Zariski, Algebraic Surfaces, 2nd suppl. ed., Ergebnisse 61, Springer-Verlag, 1971.

Daniel C. Cohen

Department of Mathematics

Louisiana State Univeristy

Baton Rouge, LA 70803 USA

e-mail: cohen@math.lsu.edu
Alexander I. Suciu

Department of Mathematics

Northeastern Univeristy

Boston, MA 02115 USA

e-mail: alexsuciu@neu.edu

(Received: September 9, 1996) 